\title{
Visualizing Conformation Transitions of the Lipid Flippase MurJ
}

\author{
Alvin C. Y. Kuk ${ }^{1}$, Aili Hao ${ }^{1}$, Ziqiang Guan ${ }^{1}$, and Seok-Yong Lee ${ }^{1, *}$
}

${ }^{1}$ Department of Biochemistry, Duke University Medical Center, 303 Research Drive, Durham, North Carolina 27710, USA

${ }^{*}$ Correspondence to: S.-Y. Lee.

Email: seok-yong.lee@duke.edu

Tel: 919-684-1005

Fax: 919-684-8885 


\begin{abstract}
The biosynthesis of many polysaccharides, including bacterial peptidoglycan and eukaryotic N-linked glycans, requires transport of lipid-linked oligosaccharide (LLO) precursors across the membrane by specialized flippases. MurJ is the flippase for the lipid-linked peptidoglycan precursor Lipid II, a key player in bacterial cell wall synthesis, and a target of recently discovered antibacterials. However, the flipping mechanism of LLOs including Lipid II remains poorly understood due to a dearth of structural information. Here we report crystal structures of MurJ captured in inward-closed, inward-open, inward-occluded and outward-facing conformations. Together with cysteine accessibility, mass spectrometry, and complementation studies, we elucidate the conformational transitions in MurJ that mediate lipid flipping, identified the key ion for function, and provide a framework for the development of inhibitors.
\end{abstract}




\section{Main text}

The biosynthesis of many important polysaccharides (including peptidoglycan, lipopolysaccharide, and N-linked glycans) necessitates membrane transport of oligosaccharide precursors from their cytoplasmic site of synthesis to their site of assembly outside the cytoplasm. To accomplish this task, cells utilize a translocase to attach the oligosaccharide precursor to a lipid carrier ${ }^{1-3}$, and a flippase to transport the lipid-linked oligosaccharide (LLO) across the membrane ${ }^{4}$. Such LLO transport systems are evolutionarily ancient and found across all domains of life from bacteria to humans ${ }^{5,6}$. LLO flipping is carried out by specialized transporters of the multidrug/oligosaccharidyl-lipid/polysaccharide (MOP) superfamily, which are structurally distinct from other transporter superfamilies such as the major facilitator superfamily. MOP transporters mediate the export of numerous molecules of physiological or pharmacological importance, including LLO precursors for the biosynthesis of bacterial cell wall (MurJ, MVF family) ${ }^{7-9}$, cell surface polysaccharides (Wzx, PST family) ${ }^{10-13}$, eukaryotic Nlinked glycosylation (Rft1, OLF family) ${ }^{14-17}$, as well as expulsion of drug molecules (MATE family) ${ }^{18}$.

Lipid II, undecaprenyl-diphosphate-MurNAc-pentapeptide-GlcNAc, is an essential lipidlinked peptidoglycan precursor that is large $(\sim 1900 \mathrm{Da})$, highly flexible ( $\sim 70$ rotatable bonds) and negatively charged (Fig. 1a). It is synthesized by the combined activities of MraY and MurG, which transfer phospho-MurNAc-pentapeptide and GlcNAc moieties to the lipid carrier undecaprenyl-phosphate (Fig. 1b) ${ }^{1,19}$. The cytoplasmic-facing Lipid II is flipped to the periplasmic side of the membrane by a Lipid II flippase, after which the GlcNAc-MurNAcpentapeptide moiety is incorporated into peptidoglycan and the lipid carrier is recycled (Fig. 1b). Recent studies provide strong evidence that MurJ, a MOP superfamily member, is the long- 
sought-after Lipid II flippase ${ }^{7-9}$. Consistent with this idea, in vitro binding of Lipid II to MurJ from Escherichia coli has recently been demonstrated by native mass spectrometry ${ }^{20}$. Studies also show that MurJ is a key player in bacterial cell division ${ }^{21}$ and is the target of newlydiscovered inhibitors, such as the humimycins ${ }^{22,23}$ and the phage M lysis protein ${ }^{24}$, both of which inhibit MurJ function to exert their bactericidal effects. Beyond bacterial physiology and antibiotic development, MurJ is important as a prototype LLO flippase and provides a framework for understanding the LLO flipping mechanism.

We previously determined a crystal structure of MurJ from Thermosipho africanus (MurJ $\mathrm{J}_{\mathrm{TA}}$ ), which exhibits an inward-facing conformation ${ }^{25}$. MurJ consists of 14 transmembrane helices (TMs), of which the first 12 are organized into two 6-TM bundles termed the N-lobe (TMs 1-6) and C-lobe (TMs 7-12) (Fig. 1c) ${ }^{25}$. The N- and C-lobes of MOP superfamily transporters are related by pseudo-twofold rotational symmetry with axis normal to the membrane, although in this MurJ structure the symmetry is notably distorted between TM 1 and 7, as well as between TM 2 and TM 8 (Fig. 1d) ${ }^{25}$. The N- and C-lobe enclose a central cavity that is large and highly cationic, properties consistent with Lipid II transport. TMs 13-14, not part of the core transport domain, form a hydrophobic groove that connects to the central cavity through a membrane portal. Based on this structure and previously reported chemical genetics data ${ }^{7}$, we proposed that MurJ transports Lipid II from the inner to the outer leaflet of the membrane using an alternating-access mechanism ${ }^{25}$ distinct from what has been proposed for other lipid transporters ${ }^{26,27}$.

Transporters which utilize an alternating access mechanism typically undergo multiple conformational states, including inward-facing open, inward-facing occluded, outward-facing occluded, and outward-facing open ${ }^{28,29}$. Because only one state has been previously visualized 
in MurJ, the design principles and conformation transitions of flippases in the MOP superfamily are poorly understood ${ }^{25}$. Furthermore, the questions of whether MurJ is an ion-coupled transporter and which ion(s) are involved remain unresolved. While some drug exporters in the MOP superfamily have been characterized to be $\mathrm{Na}^{+}$or $\mathrm{H}^{+}$coupled ${ }^{12,30-32}$, our high-resolution inward-facing MurJ structure did not reveal a cation that could be important for transport ${ }^{25}$. It has been shown that MurJ activity is dependent on membrane potential and not on a $\mathrm{H}^{+}$gradient

${ }^{33}$, but the involvement of $\mathrm{Na}^{+}$has not been ruled out. To better understand the Lipid II transport mechanism, we determined structures of MurJ in multiple stages of its transport cycle, probed the in vitro conformation of MurJ, and assessed the in vivo transport competency of MurJ mutants.

\section{Structure determination of the Lipid II flippase MurJ $\mathbf{J}_{\mathrm{TA}}$ in multiple conformations}

Because we observed a chloride ion in the central cavity of our previous crystal structure, we hypothesized that chloride might be restricting MurJ $_{\mathrm{TA}}$ in this crystal form and took precautions to remove chloride from cell lysis and protein purification buffers. With this new protein purification procedure and in the presence of Lipid II doped into the lipidic cubic phase, we obtained new crystal forms of MurJ $\mathrm{J}_{\mathrm{TA}}$ (Extended Data Fig. 1a). We determined four new crystal structures of MurJ $_{\mathrm{TA}}$ : one structure in an outward-facing conformation to $1.8 \AA$ resolution, and three structures in inward-facing conformations distinct from the published structure, resolved to 3.2, 3.0, and 2.6 A respectively (Fig. 1e). We refined the structures to good geometry and minimal clashes (Extended Data Table 1). The final electron density (Extended Data Fig. 1b) allowed almost all residues to be built unambiguously except for a few residues at the termini. 


\section{Inward-facing structures reveal a lateral-gating mechanism}

Superposition of all the inward-facing structures show that they align well at the periplasmic end of the protein (Fig. 2a), which highlights the importance of the periplasmic gate in stabilizing the inward-facing state. This gate is formed by hydrogen-bond interactions between the N-lobe and C-lobe (Fig. 2b). In contrast to the periplasmic gate, the structures diverge substantially on the cytoplasmic side (Fig. 2c-d). Coordinated rearrangement of TM 1 (blue), TM 8 (green), and the loop between TM 4 and TM 5 (TM 4-5 loop) regulates the size of the membrane portal (Fig. 2c-d). Based on the conformation at these regions and the size of the membrane portal, we assigned two of the new structures as inward-closed and inward-open. The C $\alpha$-C $\alpha$ distance between Ser11 (TM 1) and Ser267 (TM 8) increased from $8.0 \AA$ in the closed structure to $17.4 \AA$ in the open structure. TM 1 is bent outwards by almost $40^{\circ}$ in the open structure about the invariant Gly21, coordinated by rearrangement of the TM 4-5 loop and unwinding of the cytoplasmic ends of TM 4 and TM 5 (Fig. 2d). The conserved Phe151 (Phe157 in E. coli MurJ) at the TM 4-5 loop provides leverage to bend TM 1 out into the membrane. TM 8 , which contains several non- $\alpha$ helical ( $\pi$ helical or $3{ }_{10}$ helical) elements, undergoes dynamic changes that allow hinge bending and register shift (Fig. 2c). Because Lipid II is believed to access the central cavity of MurJ through the portal between TM 1 and TM $8^{25}$, these changes in the portal could regulate the entry of the large Lipid II headgroup into the central cavity via a lateral-gating mechanism. Residues in TM 1 (Arg18 and Gly21) as well as those in the TM 4-5 loop (Leu145, Asn146, Phe151, and Pro158) are conserved in MurJ but not in Multidrug And Toxic compound Extrusion (MATE) drug efflux pumps in the same superfamily (Extended Data 
Fig. 2), suggesting the lateral gate to be a specific adaptation for flippase function and thus a design principle for a LLO flippase.

To probe the in-vitro conformational states of MurJ $\mathrm{J}_{\mathrm{TA}}$, we designed single cysteine mutants at the cytoplasmic and periplasmic gates. Because MurJ $\mathrm{T}_{\mathrm{TA}}$ has no endogenous cysteines, these mutants would manifest an upward shift in mass if the cysteine was accessible when probed by PEG-maleimide. We observed that single cysteine mutants at the cytoplasmic gate were much more accessible to PEG-maleimide than those at the periplasmic gate, suggesting that Mur $\mathrm{J}_{\mathrm{TA}}$ predominantly adopts inward-facing conformations in detergent micelles (Extended Data Fig. 3). We also performed the experiments at an elevated temperature $\left(60^{\circ} \mathrm{C}\right)$ because $\operatorname{Mur} \mathrm{J}_{\mathrm{TA}}$ is from a hyperthermophile bacterium. We observed some changes in the accessibility profile at $60^{\circ} \mathrm{C}$ including reduction of cysteine accessibility at the residues comprising the cytoplasmic gate on TM 8 (Thr270, Ser274 and Ser277), indicating that MurJ $\mathrm{J}_{\mathrm{TA}}$ in detergent micelles is sampling multiple conformations. 


\section{Asymmetric inward-occluded conformation of $\mathbf{M u r} \mathbf{J}_{\mathrm{TA}}$}

One of the inward-facing structures exhibited a partially dilated membrane portal but was otherwise divergent from the rest of the inward-facing structures (Fig. 3a). Specifically, the Clobe is bent with the cytoplasmic section swung $\sim 15^{\circ}$ towards the N-lobe. Conserved residues Pro260, Pro300 and Gly340 serve as hinges on TMs 8, 9 and 10, respectively. In contrast, rearrangements in the N-lobe are restricted to the middle section of TM 2, which also bends inwards towards the central cavity. These conformational changes appear to be aided by the flexible cytoplasmic half of TM 8, which assumes a distinctly non- $\alpha$-helical conformation, as well as the broken helix in TM 2 formed by the G/A-E-G-A motif that is conserved in Gramnegative MurJ sequences (Fig. 3b). Together these changes position Glu57 from the G/A-E-G-A motif into proximity $(\sim 4 \AA$ ) of Arg352 on TM 10 (or Lys368 one helical-turn up in E. coli MurJ), forming a thin gate between the N-lobe and C-lobe (Fig. 3a-b). Notably, we observed unmodeled electron density peaks at the portal and at the central cavity above the thin gate (Fig. 3c). Although we cannot unambiguously assign the electron density to Lipid II, we hypothesized that this Glu57-Arg352 thin gate could occlude the flexible Lipid II molecule into the cavity prior to outward transition. We performed in-silico docking and molecular dynamics (MD) simulation of the docked MurJ-Lipid II complex in a hydrated phospholipid bilayer system, which shows that the inward-occluded cavity could fit the Lipid II headgroup above the thin gate (Extended Data Fig. 4a-c). To test the importance of this thin gate to MurJ function, we mutated Arg352 to either Ala or Gln, both of which led to loss of MurJ $\mathrm{J}_{\mathrm{TA}}$ function without loss of expression (Extended Data Fig. 5 and 6). Together with previous mutagenesis studies which showed that Glu57 is essential for MurJ function ${ }^{25}$, this suggests that the thin gate is important for occluding the Lipid II headgroup from the cytoplasm (Extended Data Fig. 4d). Based on the 
abovementioned conformational changes, presence of the thin gate, and the unmodeled electron density, we assign this structure as an inward-occluded state of MurJ. Inward-to-outward transition appears to occur asymmetrically with a larger rotation of the C-lobe for thin gate formation than the N-lobe.

\section{Outward-facing conformation of MurJTA}

Our high-resolution outward-facing structure allows for structural comparison with the inward-facing occluded structure to determine the conformational changes that could drive outward transition. The outward-facing cavity was substantially shallower and narrower than the inward-facing cavity, and thus cannot accommodate the head group of Lipid II. This suggests that our outward facing structure represents a state in which the head group of Lipid II is released into the periplasm, consistent with the idea that cavity shrinkage could be a mechanism to displace substrate into the periplasm ${ }^{32,34}$ (Fig. 4a). The inward-to-outward state transition is associated with a rotation of the $\mathrm{N}$ - and $\mathrm{C}$-lobes resulting in cytoplasmic gate closure and periplasmic gate opening (Fig. 4b). The N-lobe rotated by $\sim 15^{\circ}$ from the inward-occluded structure while the C-lobe rotated by only $\sim 7.5^{\circ}$, possibly because the latter has swung inwards in the asymmetric occluded structure (Fig. 4b). The fulcrum of this rotation was located at the TM 7 bridging the $\mathrm{N}$ - and C-lobes, which was bent by $90^{\circ}$ in the inward-occluded structure but straightened in the outward-facing structure. This results in concomitant lowering of the TM 6-7 loop, which is amphipathic and partially embedded in the cytoplasmic leaflet of the membrane as it loops around the N-lobe. We postulate that tension from TM 7 straightening would lower the TM 6-7 loop in the membrane, providing the force required for N-lobe rotation and closure of 
the cytoplasmic gate. TM 1 also straightens from its bent conformation in the inward-occluded structure, closing the lateral membrane portal (Fig. 4c). We observed unmodeled density in the hydrophobic groove leading into the outward-facing cavity, which could be the binding site of the undecaprenyl lipid tail of Lipid II while the headgroup is in the outward-facing cavity (Extended Data Fig. 7). Below the shallow outward-facing central cavity is the cytoplasmic gate which is $\sim 20 \AA$ thick and formed by TMs 2,8 and 10 (Fig. 4d). This gate is stabilized by hydrogen bonds between TM 2 and TM 10, again involving Arg352. Notably, Arg352 forms the thin gate with Glu57 in the inward-occluded structure, where it interacts with Ser61, Ser62, and the backbone carbonyl of Gly58 in the G/A-E-G-A helix break, suggesting that Arg352 plays a key role in conformational transition from the inward-facing to the outward-facing states.

\section{The sodium site in $\mathbf{M u r J}_{\mathrm{TA}}$}

We noticed a strong Fo - Fc omit electron density peak $(\sim 19 \sigma)$ in the C-lobe between TMs 7, 11, and 12 (Fig. 5a). The coordination distances were within the expected range for $\mathrm{Na}^{+}$ (2.3 to $2.5 \AA$ ) in our $1.8 \AA$-resolution-structure and the coordination geometry is most consistent with $\mathrm{Na}^{+}$, as determined by the CheckMyMetal server ${ }^{35}$. Also, $\mathrm{Na}^{+}$is the only cation present in one of the crystallization conditions that yielded the outward-facing state (see Materials and Methods). Based on these criteria, we assign this peak as $\mathrm{Na}^{+}$. Sodium is coordinated in trigonal bipyramidal geometry, with an equatorial plane formed by Asp235, Asn374, Val390 (backbone carbonyl) and the axial positions are occupied by Thr394 and Asp378. Asp235 is the only $\mathrm{Na}^{+}$ coordinating residue from TM 7; the others are found in either TM 11 or TM 12. In light of this discovery, we re-analyzed all the other MurJ structures and found $\mathrm{Na}^{+}$bound to the inward- 
occluded and inward-closed structures as well (Fig. 5b). The $\mathrm{Na}^{+}$bound structures exhibited different degrees of TM 7 straightening, resulting in concomitant lowering of the N-terminal half of TM 7 and the TM 6-7 loop relative to the $\mathrm{Na}^{+}$site, suggesting that $\mathrm{Na}^{+}$-induced conformational change at Asp235 could be propagated down TM 7 (Fig. 5c). The outwardfacing structure displays the most straightening of TM 7 and the most optimal $\mathrm{Na}^{+}$coordination geometry (Extended Data Table 2).

Guided by our structures, we performed mutagenesis on the sodium-coordinating residues and assayed their effect on MurJ $\mathrm{J}_{\mathrm{TA}}$ function as previously described ${ }^{8,25}$. None of the mutants at the equatorial positions were able to complement, whereas certain substitutions at axial positions were tolerated (Fig. 5d). We note that the mutants failing to complement also failed to express based on the analysis of the total membrane fraction by Western blot (Extended Data Fig. 6), suggesting that substitutions at the sodium site would perturb protein folding. We next tested whether sodium could induce conformation changes in $\mathrm{MurJ}_{\mathrm{TA}}$ by an in vitro cysteine accessibility experiment. Because MurJ $\mathrm{J}_{\mathrm{TA}}$ has no endogenous cysteines, we mutated single residues at the periplasmic gate into cysteines and probed the accessibility of purified mutant proteins in buffer containing either $\mathrm{Na}^{+}$or N-methyl-D-glucamine (Extended Data Fig. 8). Positions Ala29, Ser240 and Ile247 experienced a modest 1.5 to 2.5-fold increase in accessibility, but not other positions at or near the periplasmic gate (Met46, Ser113, Ser248). Taken together, our data show that the $\mathrm{Na}^{+}$site is important for MurJ $J_{\text {TA }}$ function or folding.

\section{State-dependent changes in the central cavity}


The central cavity in MurJ $\mathrm{J}_{\mathrm{TA}}$ undergoes state-dependent changes in its size and position with respect to the membrane. The cavity was largest in the inward-open state, was located closer to the periplasmic side in the inward-occluded state, and resembled a narrow crevice in the outward-facing state (Fig. 6a). We observed systematic rearrangement of Arg24, Asp25, and Arg255 (Arg270 in E. coli MurJ) in the cavity between the different crystal structures (Fig. 6b). These three residues are conserved with Arg24 being invariant. Both Arg24/Arg255 are essential ${ }^{36,37}$, while Asp25 is intolerant of certain substitutions ${ }^{38}$. This Arg24-Asp25-Arg255 triad is held in position by Asn162, Gln251, and a chloride ion in the inward-closed state. Arg255 is released from Gln251 in the inward-open state and swivels down, possibly to capture the diphosphate moiety of Lipid II that enters through the portal. In the inward-occluded state, Arg24 and Arg255 are brought into unusual proximity (just $2.9 \AA$ apart at the closest) on top of the unmodeled electron density. Since such close contact between two arginine side chains would experience unfavorable repulsion, we reasoned that they could be stabilized by electrostatic interactions with Asp25 and the diphosphate moiety of Lipid II. The periplasmic gate opens in the outward conformation, disengaging Arg255 and allowing substrate release. Because of their conserved and essential nature, as well as their coordinated rearrangement at the apex of the inward-facing cavity in proximity to the unmodeled electron density, we suggest Arg24-Asp25-Arg255 to be the putative substrate-binding triad.

We next tested whether these residues are important for MurJ $_{\mathrm{TA}}$ function by performing a mass spectrometry assay for cellular Lipid II accumulation (Extended Data Fig. 9). Since MurJ is essential in E. coli, depletion of MurJ results in defect in cell growth and eventually cell death ${ }^{8}$. Expression of wild-type MurJ $\mathrm{J}_{\mathrm{TA}}$ rescued cell growth but not mutants R24A and R255A (Extended Data Fig. 9a), consistent with our previous results indicating that these two mutants 
are non-functional ${ }^{25}$. Because it has been reported that Lipid II accumulates when MurJ activity is abolished ${ }^{33,39}$, we directly examined cellular Lipid II levels in these cells. Lipid II levels were approximately five-fold higher in cells expressing the R24A or R255A MurJ $\mathrm{TA}_{\mathrm{TA}}$ mutants as compared to that in wild-type MurJ $\mathrm{T}_{\mathrm{TA}}$ (Extended Data Fig. 9b), which is consistent with importance of these residues in Lipid II transport.

\section{Discussion}

We determined the conformational landscape of the lipid flippase MurJ, and our analyses suggest that MurJ undergoes an ordered trajectory through its transport cycle (Fig. 6c). Our study highlights the importance of dynamic conformation changes to the mechanism of Lipid II flipping, which could be a principle for LLO flipping by LLO flippases in general. In particular, TM 8 undergoes dynamic conformational changes through transient $\pi$ or 310 helical elements throughout the transport cycle. These dynamic helical secondary structure changes were proposed to be important for gating of Transient Receptor Potential ion channels ${ }^{40}$, but their presence in our MurJ $_{\mathrm{TA}}$ structures suggest that they are more universal for membrane transport proteins than were previously recognized.

The outward-facing structure of MurJ $_{\mathrm{TA}}$ indicates importance of the cytoplasmic gate in occluding access to the outward-facing cavity from the cytoplasm. Because we observed substantial divergence at the cytoplasmic gate among the inward-facing structures, dynamic conformation changes at this gate appear to be important for MurJ function. Consistent with this finding, resistance mutants to the humimycins, lipopeptides with antibacterial activity against some Gram-positive pathogens including methicillin-resistant Staphylococcus aureus ${ }^{22}$, map 
mostly to the cytoplasmic side of MurJ below the putative lipid II headgroup binding site ${ }^{23}$. While the mechanism of inhibition by humimycins is not currently known, it is conceivable that they could disrupt closure of the cytoplasmic gate and thus trap MurJ in one of the inward-facing conformations.

These structures of MurJ $_{\mathrm{TA}}$ also demonstrate the importance of dynamic TM 7 rearrangement for inward to outward transition. Resistant mutants to the levivirus M lysis protein $\left(\mathrm{Lys}^{\mathrm{M}}\right)$, which induces bacterial cell lysis by inhibition of MurJ function, was previously mapped to the TM 2-TM 7 region that is facing outside towards the membrane ${ }^{24}$. Cysteines positioned in the cavity displayed increased MTSES accessibility in cells upon Lys ${ }^{\mathrm{M}}$ treatment, suggesting stabilization of the outward-facing state upon Lys $^{\mathrm{M}}$ binding ${ }^{24,33}$, consistent with the role of TM7 in controlling the conformational state of MurJ. As exemplified by humimycins and Lys $^{\mathrm{M}}$, our study provides a framework for understanding how the conformational dynamics of a LLO flippase could be exploited by natural product inhibitors for antibiotic targeting. 


\section{References}

1. Chung, B.C. et al. Crystal structure of MraY, an essential membrane enzyme for bacterial cell wall synthesis. Science 341, 1012-1016 (2013).

2. Chung, B.C. et al. Structural insights into inhibition of lipid I production in bacterial cell wall synthesis. Nature 533, 557-560 (2016).

3. Yoo, J. et al. GlcNAc-1-P-transferase-tunicamycin complex structure reveals basis for inhibition of N-glycosylation. Nat Struct Mol Biol 25, 217-224 (2018).

4. Hvorup, R.N. et al. The multidrug/oligosaccharidyl-lipid/polysaccharide (MOP) exporter superfamily. Eur J Biochem 270, 799-813 (2003).

5. Ruiz, N. Lipid Flippases for Bacterial Peptidoglycan Biosynthesis. Lipid Insights 8, 2131 (2015).

6. Sanyal, S. \& Menon, A.K. Flipping lipids: why an' what's the reason for? ACS Chem Biol 4, 895-909 (2009).

7. Sham, L.-T. et al. MurJ is the flippase of lipid-linked precursors for peptidoglycan biogenesis. Science 345, 220-222 (2014).

8. Ruiz, N. Bioinformatics identification of MurJ (MviN) as the peptidoglycan lipid II flippase in Escherichia coli. Proc Natl Acad Sci U S A 105, 15553-7 (2008).

9. Inoue, A. et al. Involvement of an essential gene, mviN, in murein synthesis in Escherichia coli. J Bacteriol 190, 7298-301 (2008).

10. Liu, D., Cole, R.A. \& Reeves, P.R. An O-antigen processing function for Wzx (RfbX): a promising candidate for O-unit flippase. J Bacteriol 178, 2102-7 (1996).

11. Islam, S.T. et al. A cationic lumen in the Wzx flippase mediates anionic O-antigen subunit translocation in Pseudomonas aeruginosa PAO1. Mol Microbiol 84, 1165-76 (2012).

12. Islam, S.T. et al. Proton-dependent gating and proton uptake by Wzx support O-antigensubunit antiport across the bacterial inner membrane. MBio 4, e00678-13 (2013).

13. Liu, M.A., Morris, P. \& Reeves, P.R. Wzx flippases exhibiting complex O-unit preferences require a new model for WzX-substrate interactions. Microbiologyopen, e00655 (2018).

14. Helenius, J. et al. Translocation of lipid-linked oligosaccharides across the ER membrane requires Rft1 protein. Nature 415, 447-50 (2002).

15. Frank, C.G., Sanyal, S., Rush, J.S., Waechter, C.J. \& Menon, A.K. Does Rft1 flip an Nglycan lipid precursor? Nature 454, E3-4; discussion E4-5 (2008).

16. Sanyal, S., Frank, C.G. \& Menon, A.K. Distinct flippases translocate glycerophospholipids and oligosaccharide diphosphate dolichols across the endoplasmic reticulum. Biochemistry 47, 7937-46 (2008).

17. Rush, J.S., Gao, N., Lehrman, M.A., Matveev, S. \& Waechter, C.J. Suppression of Rft1 expression does not impair the transbilayer movement of Man5GlcNAc2-P-P-dolichol in sealed microsomes from yeast. J Biol Chem 284, 19835-42 (2009).

18. Kuroda, T. \& Tsuchiya, T. Multidrug efflux transporters in the MATE family. Biochim Biophys Acta 1794, 763-8 (2009).

19. Ha, S., Walker, D., Shi, Y. \& Walker, S. The 1.9 A crystal structure of Escherichia coli MurG, a membrane-associated glycosyltransferase involved in peptidoglycan biosynthesis. Protein Sci 9, 1045-52 (2000). 
20. Bolla, J.R. et al. Direct observation of the influence of cardiolipin and antibiotics on lipid II binding to MurJ. Nat Chem 10, 363-371 (2018).

21. Monteiro, J.M. et al. Peptidoglycan synthesis drives an FtsZ-treadmilling-independent step of cytokinesis. Nature 554, 528-532 (2018).

22. Chu, J. et al. Discovery of MRSA active antibiotics using primary sequence from the human microbiome. Nat Chem Biol 12, 1004-1006 (2016).

23. Chu, J. et al. Human Microbiome Inspired Antibiotics with Improved beta-Lactam Synergy against MDR Staphylococcus aureus. ACS Infect Dis 4, 33-38 (2018).

24. Chamakura, K.R. et al. A viral protein antibiotic inhibits lipid II flippase activity. Nat Microbiol 2, 1480-1484 (2017).

25. Kuk, A.C., Mashalidis, E.H. \& Lee, S.Y. Crystal structure of the MOP flippase MurJ in an inward-facing conformation. Nat Struct Mol Biol 24, 171-176 (2017).

26. Brunner, J.D., Lim, N.K., Schenck, S., Duerst, A. \& Dutzler, R. X-ray structure of a calcium-activated TMEM16 lipid scramblase. Nature 516, 207-12 (2014).

27. Perez, C. et al. Structure and mechanism of an active lipid-linked oligosaccharide flippase. Nature 524, 433-8 (2015).

28. Boudker, O. \& Verdon, G. Structural perspectives on secondary active transporters. Trends Pharmacol Sci 31, 418-26 (2010).

29. Hirschi, M., Johnson, Z.L. \& Lee, S.Y. Visualizing multistep elevator-like transitions of a nucleoside transporter. Nature 545, 66-70 (2017).

30. Steed, P.R., Stein, R.A., Mishra, S., Goodman, M.C. \& McHaourab, H.S. Na(+)-substrate coupling in the multidrug antiporter norm probed with a spin-labeled substrate.

Biochemistry 52, 5790-9 (2013).

31. Claxton, D.P., Jagessar, K.L., Steed, P.R., Stein, R.A. \& McHaourab, H.S. Sodium and proton coupling in the conformational cycle of a MATE antiporter from Vibrio cholerae. Proc Natl Acad Sci U S A 115, E6182-e6190 (2018).

32. Miyauchi, H. et al. Structural basis for xenobiotic extrusion by eukaryotic MATE transporter. Nat Commun 8, 1633 (2017).

33. Rubino, F.A., Kumar, S., Ruiz, N., Walker, S. \& Kahne, D.E. Membrane Potential Is Required for MurJ Function. J Am Chem Soc 140, 4481-4484 (2018).

34. Tanaka, Y. et al. Structural basis for the drug extrusion mechanism by a MATE multidrug transporter. Nature 496, 247-51 (2013).

35. Zheng, H. et al. CheckMyMetal: a macromolecular metal-binding validation tool. Acta Crystallogr D Struct Biol 73, 223-233 (2017).

36. Butler, E.K., Davis, R.M., Bari, V., Nicholson, P.A. \& Ruiz, N. Structure-function analysis of MurJ reveals a solvent-exposed cavity containing residues essential for peptidoglycan biogenesis in Escherichia coli. J Bacteriol 195, 4639-49 (2013).

37. Butler, E.K., Tan, W.B., Joseph, H. \& Ruiz, N. Charge requirements of lipid II flippase activity in Escherichia coli. J Bacteriol 196, $4111-9$ (2014).

38. Zheng, S. et al. Structure and mutagenic analysis of the lipid II flippase MurJ from Escherichia coli. Proc Natl Acad Sci U S A 115, 6709-6714 (2018).

39. Qiao, Y. et al. Lipid II overproduction allows direct assay of transpeptidase inhibition by beta-lactams. Nat Chem Biol 13, 793-798 (2017).

40. Zubcevic, L. et al. Cryo-electron microscopy structure of the TRPV2 ion channel. Nat Struct Mol Biol 23, 180-186 (2016). 
41. Caffrey, M. \& Cherezov, V. Crystallizing membrane proteins using lipidic mesophases. Nat Protoc 4, 706-31 (2009).

42. Kabsch, W. XDS. Acta Crystallogr D Biol Crystallogr 66, 125-32 (2010).

43. Foadi, J. et al. Clustering procedures for the optimal selection of data sets from multiple crystals in macromolecular crystallography. Acta Crystallogr D Biol Crystallogr 69, 1617-32 (2013).

44. Evans, P.R. An introduction to data reduction: space-group determination, scaling and intensity statistics. Acta Crystallogr D Biol Crystallogr 67, 282-92 (2011).

45. Evans, P.R. \& Murshudov, G.N. How good are my data and what is the resolution? Acta Crystallogr D Biol Crystallogr 69, 1204-14 (2013).

46. Tickle, I.J., Flensburg, C., Keller, P., Paciorek, W., Sharff, A., \& Vonrhein, C., Bricogne, G. STARANISO. Cambridge, United Kingdom: Global Phasing Ltd. (2017).

47. McCoy, A.J. et al. Phaser crystallographic software. J Appl Crystallogr 40, 658-674 (2007).

48. Emsley, P., Lohkamp, B., Scott, W.G. \& Cowtan, K. Features and development of Coot. Acta Crystallogr D Biol Crystallogr 66, 486-501 (2010).

49. Afonine, P.V. et al. Towards automated crystallographic structure refinement with phenix.refine. Acta Crystallogr D Biol Crystallogr 68, 352-67 (2012).

50. Delano, W.L. The PyMol Molecular Graphics System, (DeLano Scientific, Palo Alto, CA, USA, 2002).

51. Morris, G.M. et al. AutoDock4 and AutoDockTools4: Automated docking with selective receptor flexibility. J Comput Chem 30, 2785-91 (2009).

52. Trott, O.\& Olson, A.J. AutoDock Vina: improving the speed and accuracy of docking with a new scoring function, efficient optimization, and multithreading. J Comput Chem 31, 455-61 (2010).

53. Dolinsky, T.J., Nielsen, J.E., McCammon, J.A. \& Baker, N.A. PDB2PQR: an automated pipeline for the setup of Poisson-Boltzmann electrostatics calculations. Nucleic Acids Res 32, W665-7 (2004).

54. Dolinsky, T.J. et al. PDB2PQR: expanding and upgrading automated preparation of biomolecular structures for molecular simulations. Nucleic Acids Res 35, W522-5 (2007).

55. Humphrey, W., Dalke, A. \& Schulten, K. VMD: visual molecular dynamics. J Mol Graph 14, 33-8, 27-8 (1996).

56. MacKerell, A.D. et al. All-atom empirical potential for molecular modeling and dynamics studies of proteins. J Phys Chem B 102, 3586-616 (1998).

57. Schlenkrich, M., Brickmann, J., D. MacKerell, A. \& Karplus, M. An Empirical Potential Energy Function for Phospholipids: Criteria for Parameter Optimization and Applications, 31-81 (1996).

58. Feller, S.E., Yin, D., Pastor, R.W. \& MacKerell, A.D., Jr. Molecular dynamics simulation of unsaturated lipid bilayers at low hydration: parameterization and comparison with diffraction studies. Biophys J 73, 2269-79 (1997).

59. Zoete, V., Cuendet, M.A., Grosdidier, A. \& Michielin, O. SwissParam: a fast force field generation tool for small organic molecules. J Comput Chem 32, 2359-68 (2011).

60. Phillips, J.C. et al. Scalable molecular dynamics with NAMD. J Comput Chem 26, 1781802 (2005).

61. Darden, T., York, D. \& Pedersen, L. Particle mesh Ewald: An N· $\log (\mathrm{N})$ method for Ewald sums in large systems. The Journal of Chemical Physics 98, 10089-10092 (1993). 
bioRxiv preprint doi: https://doi.org/10.1101/552745; this version posted February 19, 2019. The copyright holder for this preprint (which was not certified by peer review) is the author/funder. All rights reserved. No reuse allowed without permission.

62. Bligh, E.G. \& Dyer, W.J. A rapid method of total lipid extraction and purification. Can J Biochem Physiol 37, 911-7 (1959). 


\section{Acknowledgments}

We thank Natividad Ruiz (The Ohio State University) for sharing E. coli strains NR1154 and NR1157. We thank Ellene Mashalidis for critical manuscript reading. This work was supported by the National Institutes of Health (R01GM120594 to S.-Y. L.). Data for this study were collected at NE-CAT beamlines 24-ID-C and 24-ID-E at the Advanced Photon Source, which are funded by GM124165, RR029205, and OD021527.

\section{Author Contributions}

A.K. solved the structures of MurJ $\mathrm{JA}_{\mathrm{TA}}$, performed in vitro accessibility assay, and conducted western blot analyses under the guidance of S.-Y.L. A.H. carried out the functional complementation assays and sample preparation for lipid mass spectrometry and western blot experiments under the guidance of S.-Y.L. Z.G. performed lipid mass spectrometry experiments. A.K. and S.-Y.L. wrote the paper. All authors discussed the results and commented on the manuscript.

\section{Declaration of Interests}

The authors declare no competing interests. 

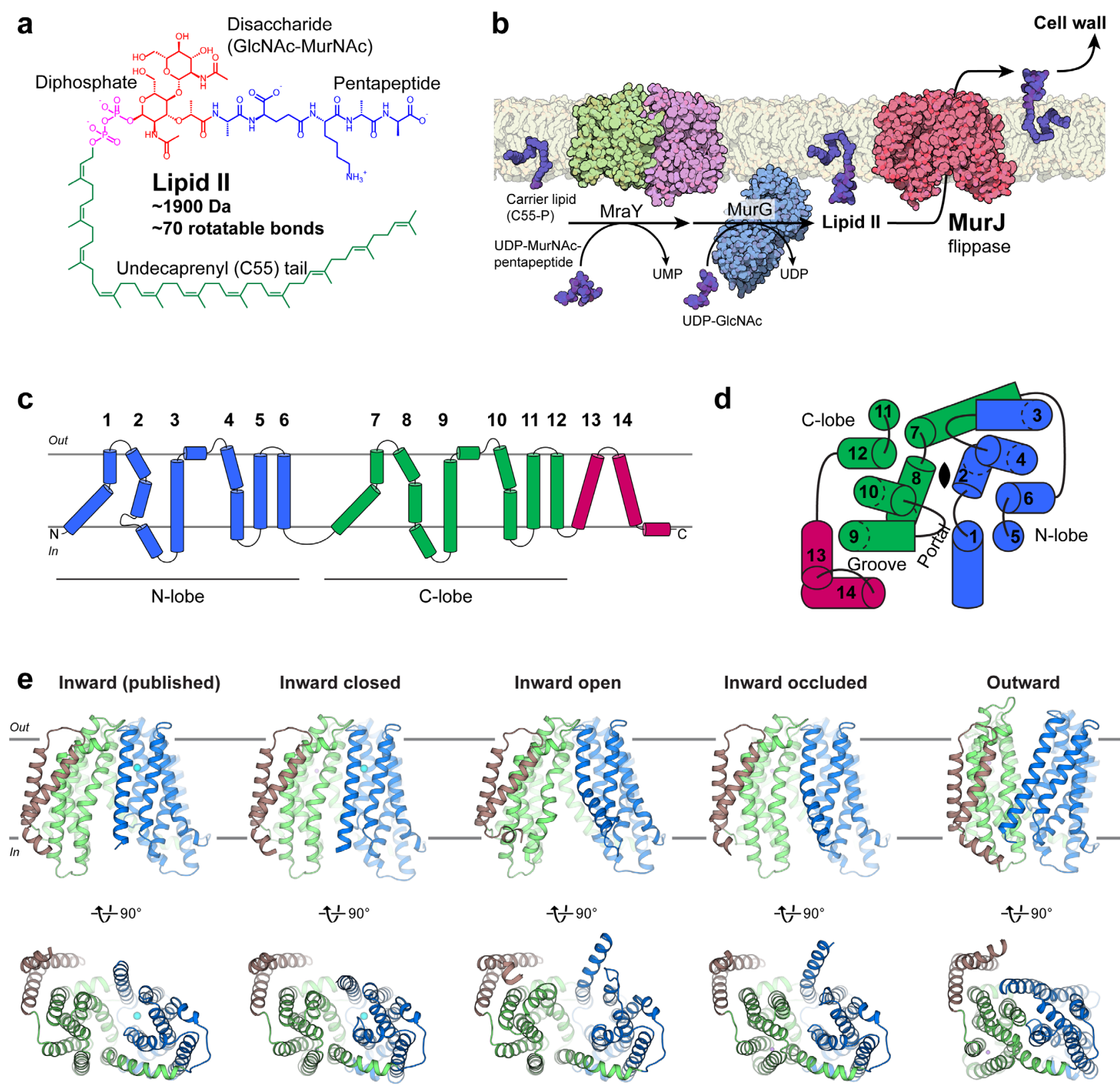

Fig. 1 | Crystal structures of the Lipid II flippase MurJta. a, Chemical structure of the peptidoglycan precursor Lipid II. b, Lipid II is synthesized by the combined transferase activities of MraY (PDB 4J72) and MurG (1F0K), then flipped across the cytoplasmic membrane by MurJ for subsequent incorporation into the cell wall. These steps constitute a prototype lipidlinked oligosaccharide (LLO) transport system. c, MurJ contains two 6-helix bundles (N-lobe, 
blue and C-lobe, green) common to the MOP superfamily and 2 additional C-terminal helices (brown). d, N-lobe and C-lobe are related by pseudo-twofold rotational symmetry normal to the membrane (top view is shown). Symmetry is distorted at TMs $1,2,7$, and 8 which enclose the central cavity. TMs 13 and 14 form a hydrophobic groove that enters the central cavity through a portal. e, Our previous crystal structure of MurJ (5T77), and new crystal structures in the inward-closed, inward-open, inward-occluded, and outward conformations (front and bottom views). Resolutions (from left) are $2.0 \AA, 3.2 \AA, 3.0 \AA, 2.6 \AA$, and $1.8 \AA$. 
a

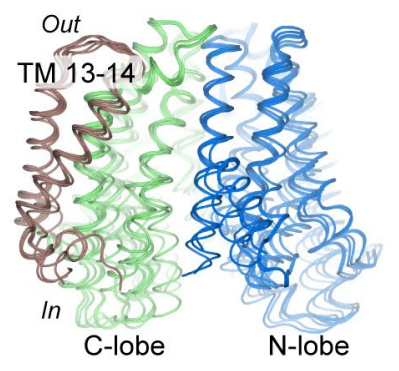

$\stackrel{\leftarrow}{\top} 45^{\circ}$ b

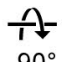
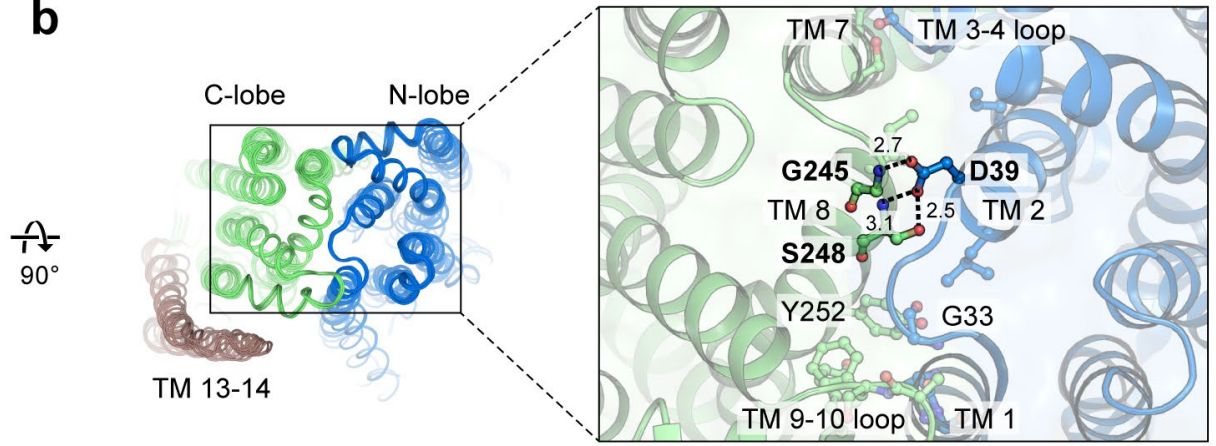
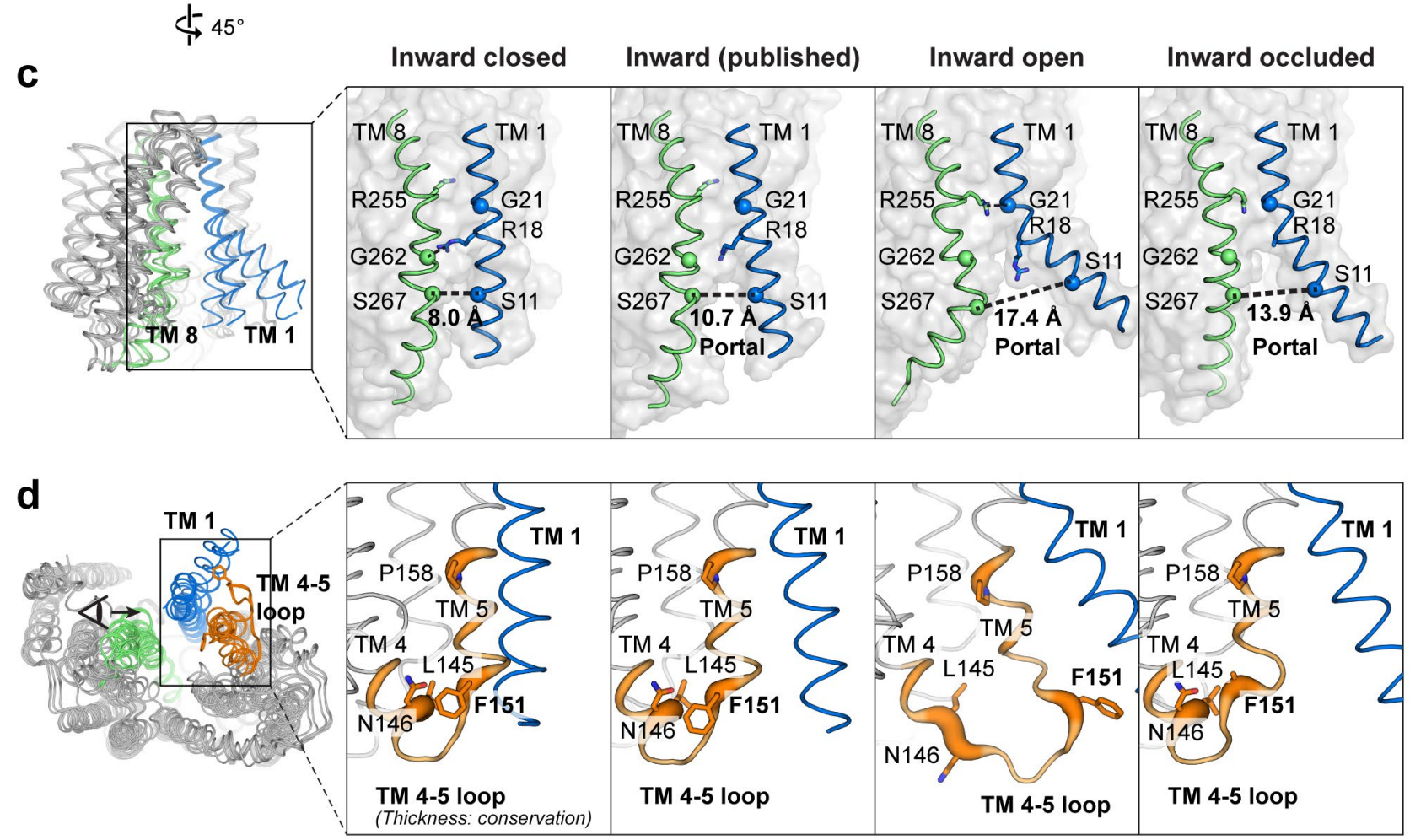

Fig. 2 Lateral gating mechanism in the inward-facing state. a, Superposition of inwardfacing structures highlights similarity at the periplasmic gate but divergence at the cytoplasmic gate. b, The periplasmic gate is stabilized by hydrogen bonds between Gly245/Ser248 and the essential Asp39. c, At the cytoplasmic side, coordinated rearrangement of TM 1 (blue) and TM 8 (green) controls portal dilation. The $\mathrm{C} \alpha-\mathrm{C} \alpha$ distance between Ser11 (TM 1) and Ser267 (TM 8) increases from $8.0 \AA$ in the inward-closed structure to $17.4 \AA$ in the inward-open structure. d, Rearrangement of the TM 4-5 loop (orange) and unwinding of the cytoplasmic ends of TMs 4/5 
could induce bending of TM 1. The conserved Phe151 (Phe157 in E. coli MurJ) provides leverage to bend TM 1 (residues 1-20) out into the membrane. TM 4-5 loop is shown in sausage representation, with thickness proportional to conservation. Sidechains of Arg18 and Phe151 were not resolved in the inward-occluded structure. 
a

Inward open

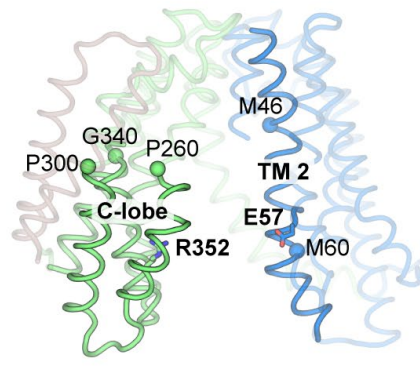

$-t+90^{\circ}$
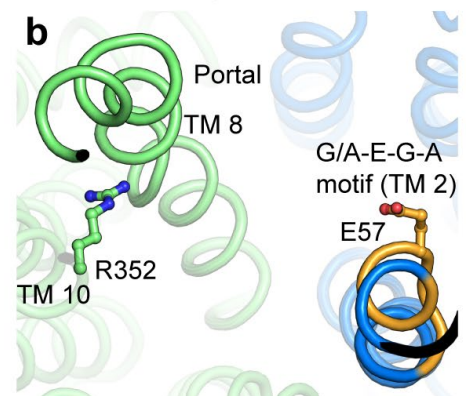

Inward occluded

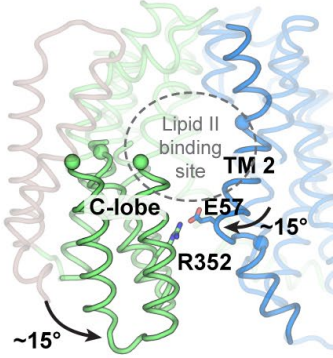

$-t+\frac{1}{5} 90^{\circ}$
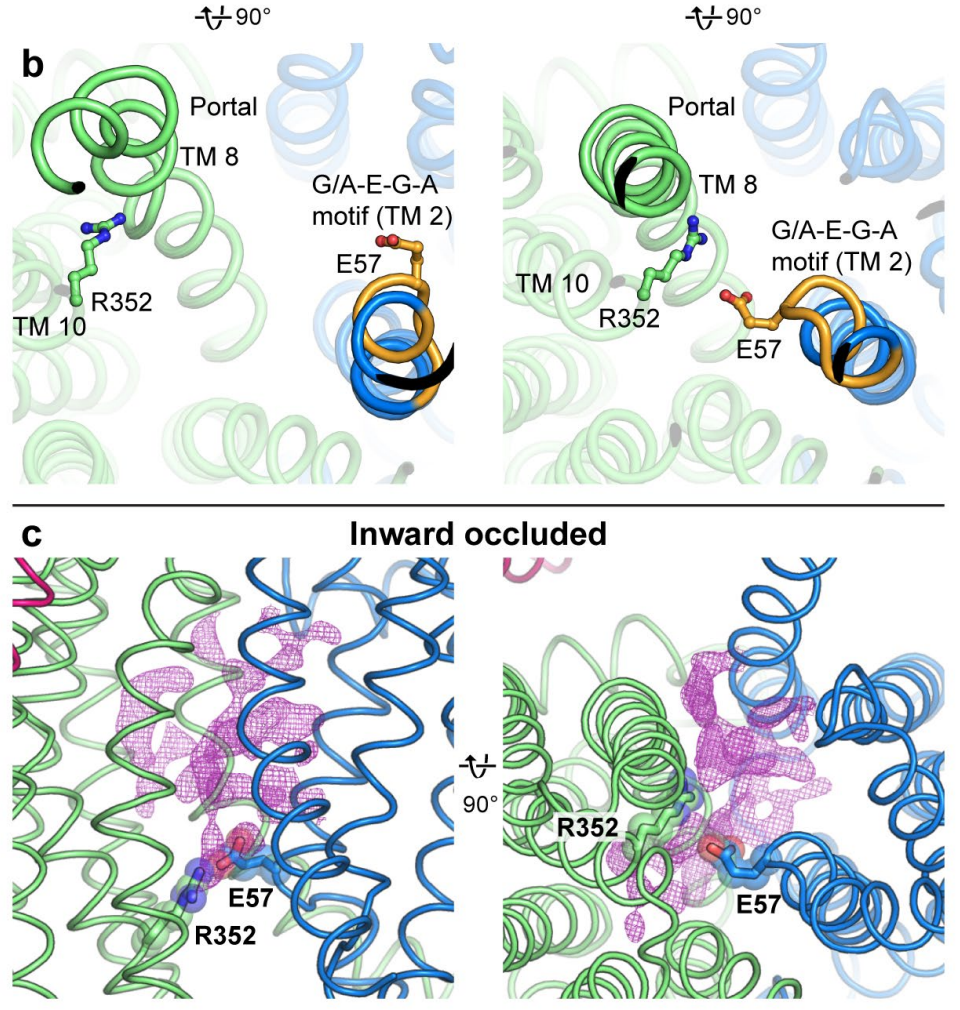

Fig. 3 | The asymmetric inward-occluded conformation of MurJta. a, Rotation of the cytoplasmic half of C-lobe by $\sim 15^{\circ}$ from the inward-open structure (left) to the inward-occluded structure (right). Conserved residues Pro260, Pro300, and Gly340 serve as hinges. At the same time, the middle segment of TM 2 also bends inwards by $\sim 15^{\circ}$. Together these motions bring Glu57 (TM 2) and Arg352 (TM 10, Lys368 in E. coli MurJ) into proximity, which form a thin gate. Most of TM 1 is hidden for clarity. b, The conserved (G/A)-E-G-A motif (orange) allows S-shaped bending of TM 2 by breaking the helix. TM 8 assumes a more $\alpha$-helical geometry (albeit still not ideal) in the inward-occluded structure than in the other inward structures. c, 

not certified by peer review) is the author/funder. All rights reserved. No reuse allowed without permission.

Unmodeled $2 \mathrm{Fo}-\mathrm{Fc}$ electron density at the portal and transport cavity of the inward-occluded structure, displayed here as purple mesh contoured to $0.8 \sigma$. 

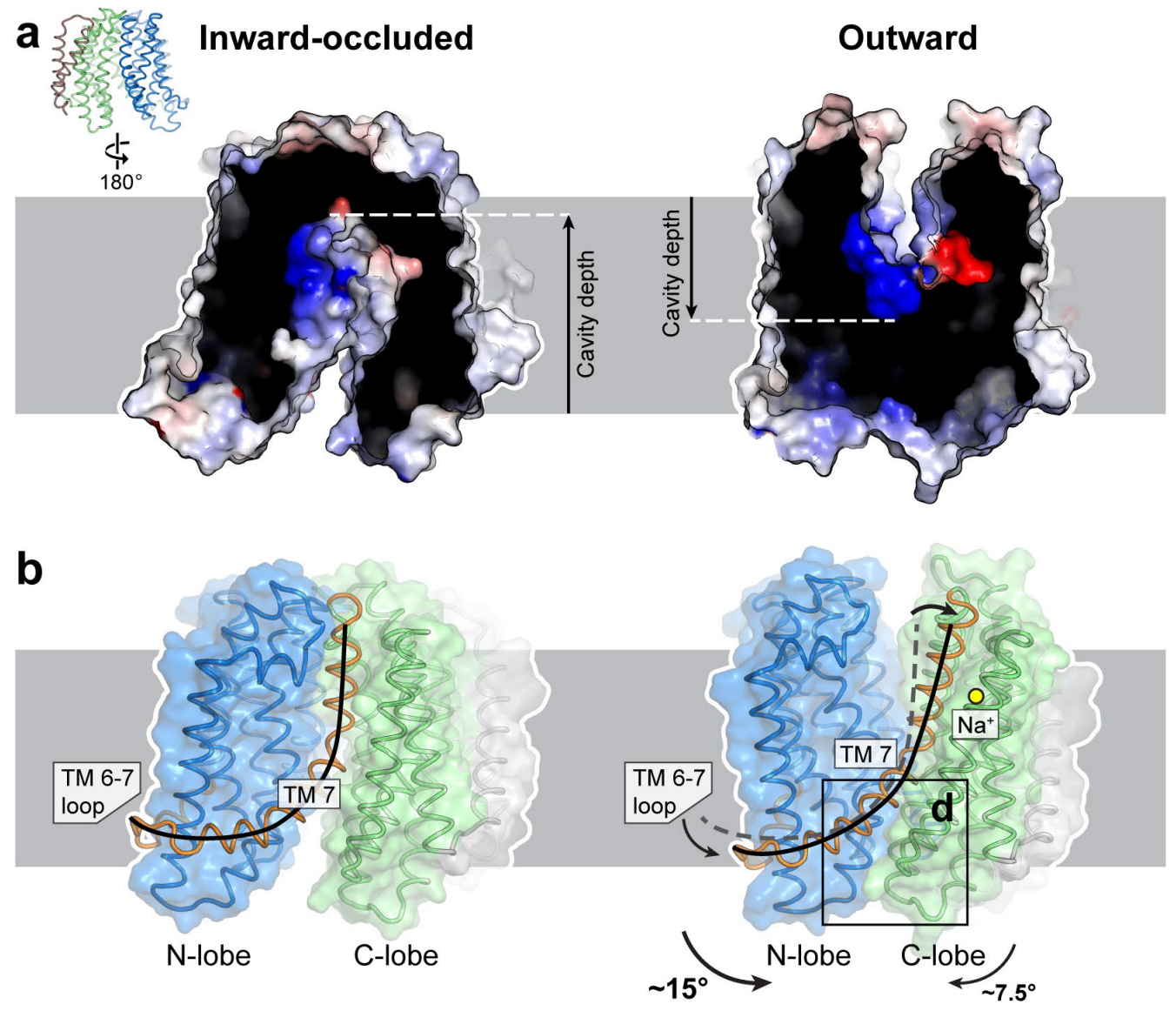

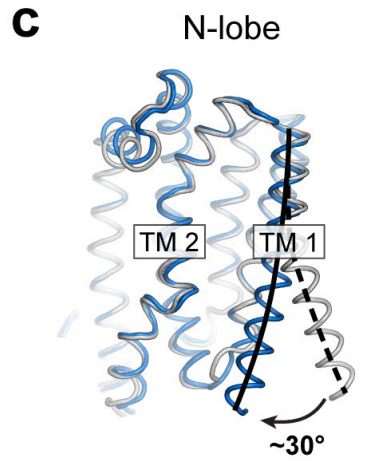

Blue: Outward

Gray: Inward-occluded

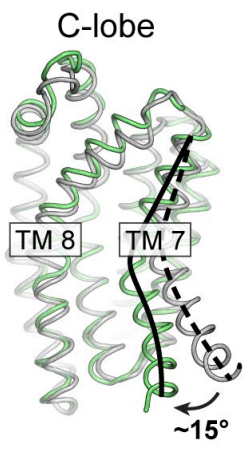

Green: Outward Gray: Inward-occluded

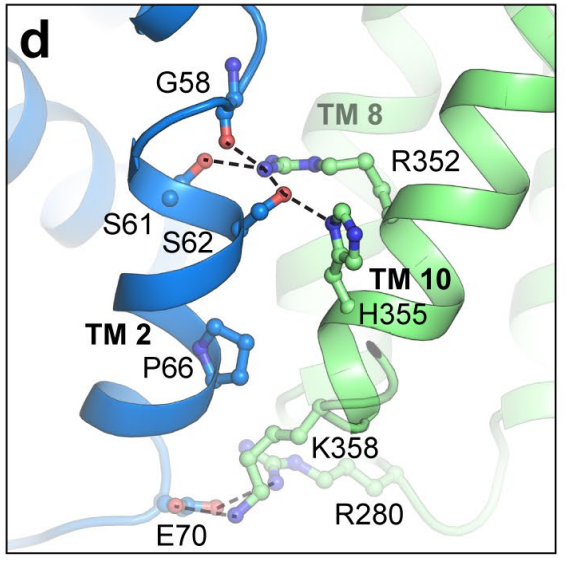

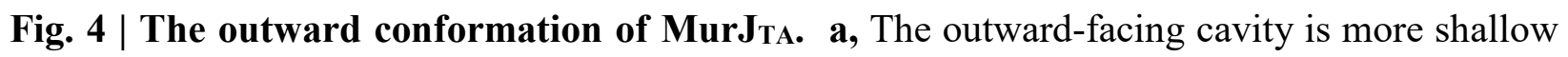
and narrow than the inward-facing cavity. b, Straightening of TM 7 and concomitant lowering of the TM 6-7 loop could close the cytoplasmic gate, allowing transition from the inwardoccluded to the outward-facing conformation. c, Alignment of $\mathrm{N}$ - and C-lobes from the inward- 
occluded conformation (gray) to those of the outward-facing conformation (in color), showing rotation of not just TM 7 but also TM 1, the latter closing the lateral membrane portal. d, The cytoplasmic gate is mainly stabilized by a hydrogen bond network between TMs 2 and 10, with less-conserved electrostatic interactions at the cytoplasmic loops. 

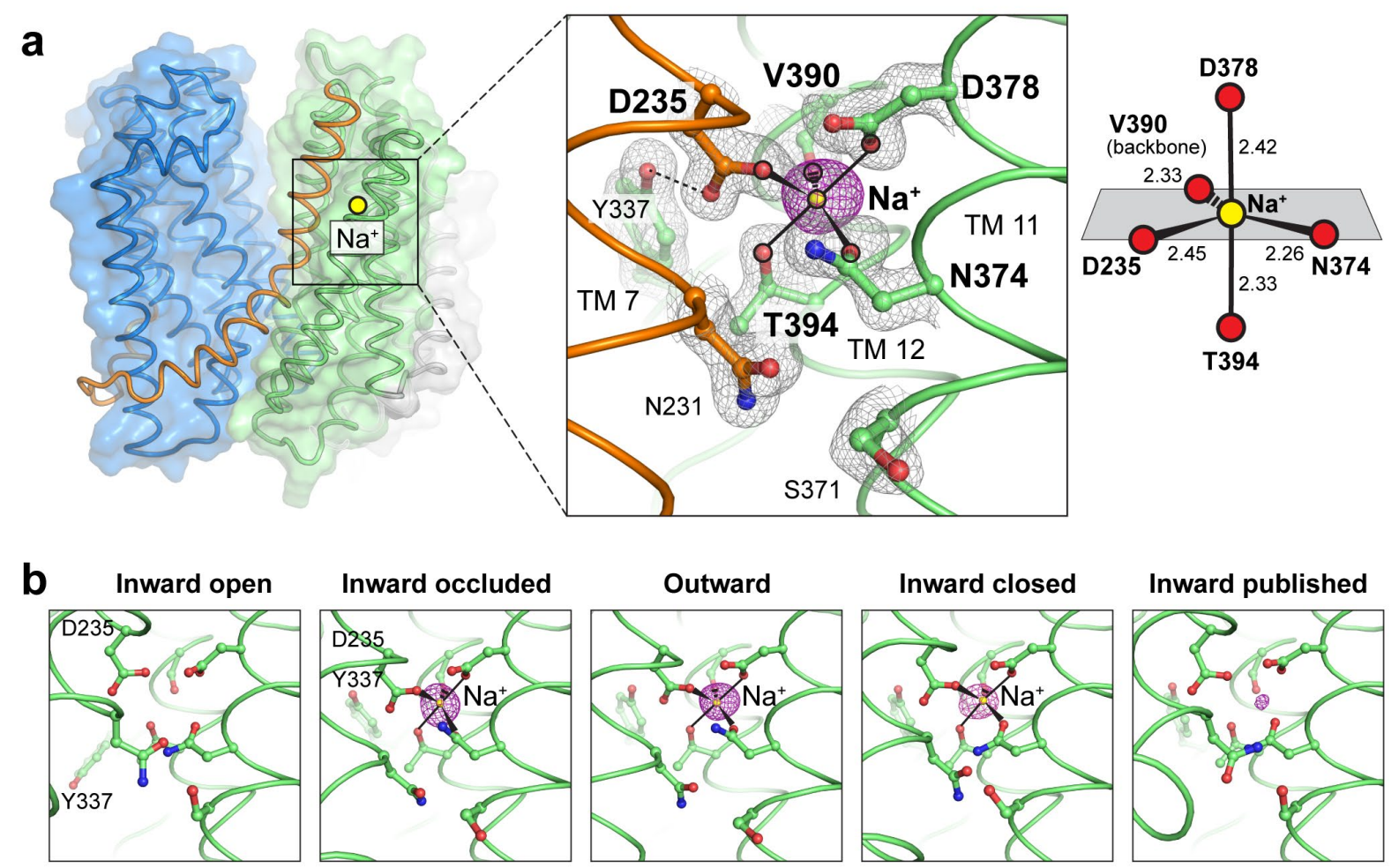

C

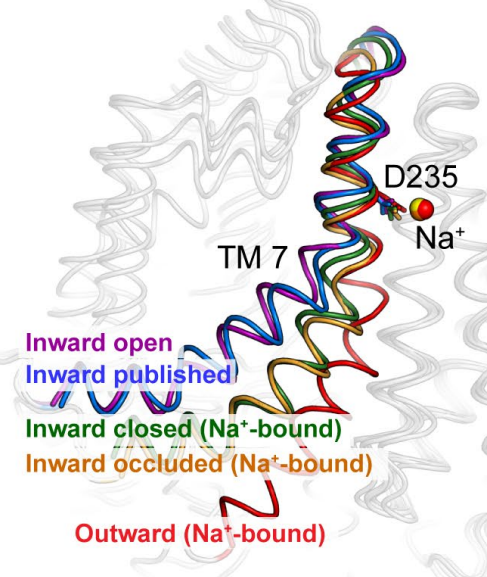

d

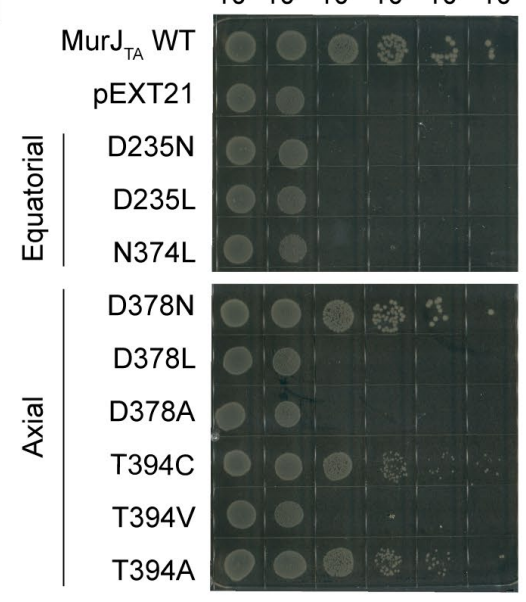

Fucose + IPTG
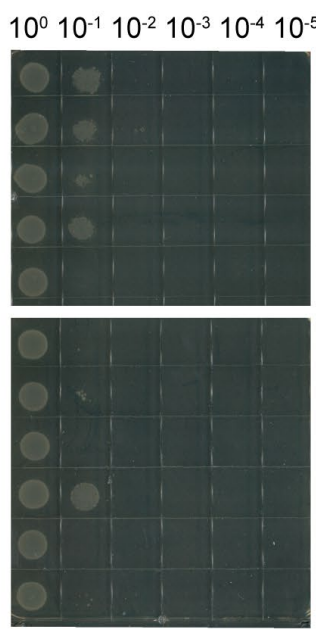

Fucose

Fig. 5 | The sodium binding site in MurJTA. a, $\mathrm{Na}^{+}$is bound in the C-lobe (TMs 7, 11, 12) coordinated by Asp235, Asn374, Asp378, Val390 (backbone carbonyl), and Thr394. Fo - Fc omit density for $\mathrm{Na}^{+}$is shown as magenta mesh contoured to $4.5 \sigma$ (peak height $\sim 19 \sigma, 1.8 \AA$ resolution). 2Fo $-\mathrm{Fc}$ omit density is shown in gray mesh. $\mathrm{Na}^{+}$is coordinated by trigonal bipyramidal geometry. $\mathbf{b}, \mathrm{Na}^{+}$was also bound in the inward-occluded and inward-closed 
structures. c, $\mathrm{Na}^{+}$-associated rearrangement could be propagated down $\mathrm{TM} 7 . \quad$ d, Complementation assay of MurJ $\mathrm{T}_{\mathrm{TA}}$ sodium site mutants in E. coli MurJ-depletion strain NR1154. Cells transformed with plasmids encoding $\operatorname{MurJ}_{\mathrm{TA}}$ (wild-type or sodium site mutant) or without insert (pEXT21) were depleted of endogenous MurJ by serial dilution on agar plates containing the anti-inducer D-fucose. MurJ $\mathrm{J}_{\mathrm{TA}}$ expression was induced by addition of IPTG. Data shown are representative of three biological replicates. Mutants D235A and N374A were previously determined to neither express nor complement ${ }^{25}$, and thus were not included here. 
a
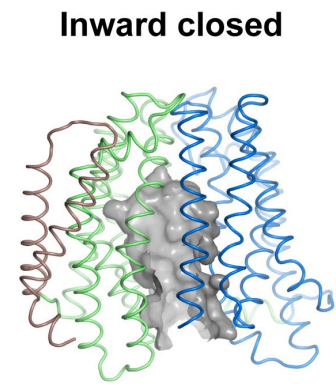

b

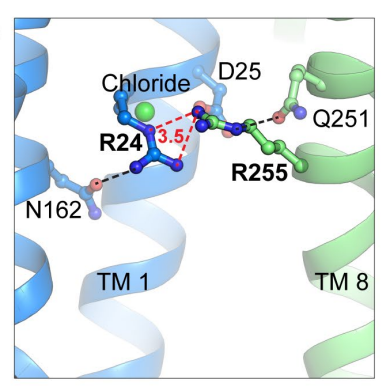

Inward open
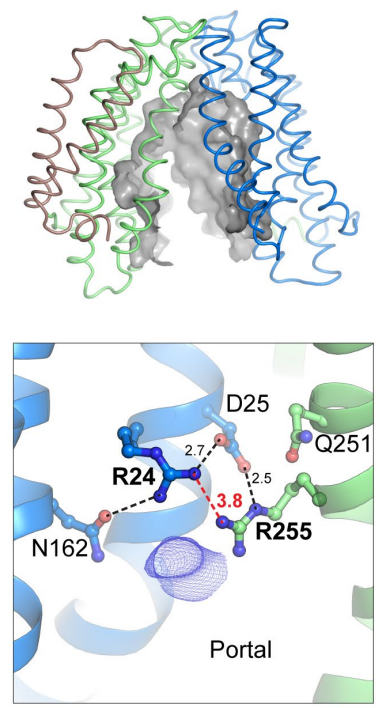

Inward open

C

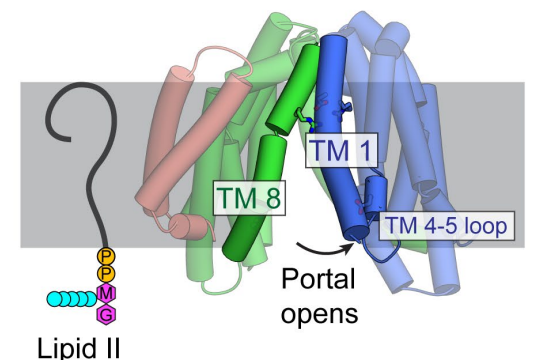

$\mathrm{Na}^{+} \ll$

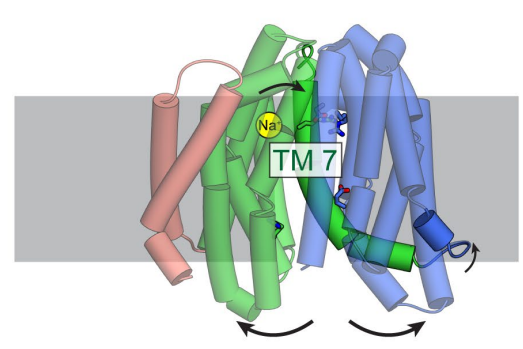

Inward closed
Inward occluded
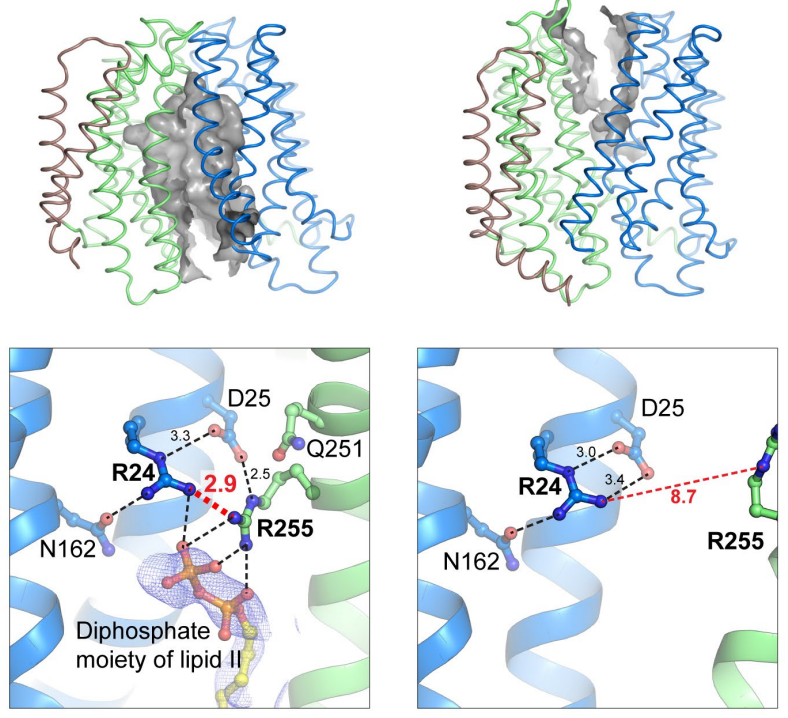

Inward occluded
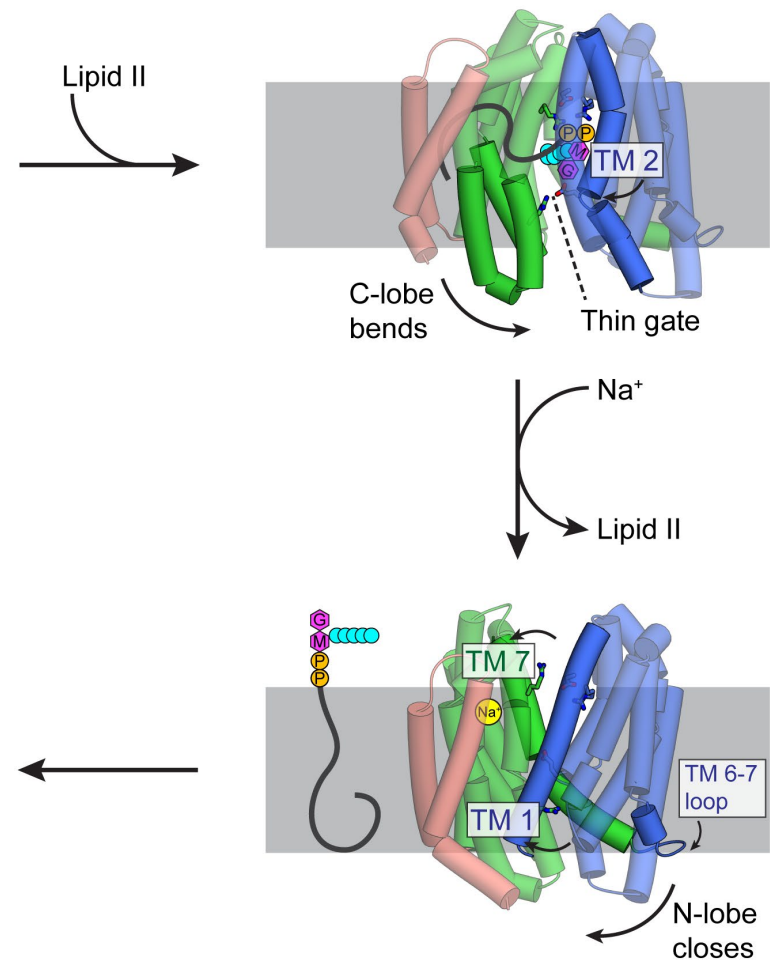

Outward

Fig. 6 | State-dependent conformational changes in the central cavity during the transport

cycle. a, The inward-facing cavity opens up in the inward-open conformation, and then extends deepest into the membrane in the inward-occluded conformation. In contrast, the outward-facing 
cavity is narrow and shallow. b, Systematic rearrangement of the Arg24-Asp25-Arg255 triad in the cavity during the transport cycle. In the inward-occluded state, Arg24 and Arg255 are brought into unusual proximity (just $2.9 \AA$ apart at the closest), with the unfavorable repulsion stabilized by electrostatic interactions with Asp25 and the diphosphate moiety of Lipid II. The lipid-diphosphate model was docked into final electron density (blue mesh, contoured to $1.0 \sigma$ ) and was never used for refinement or map calculation. c, Proposed model of the MurJ transport mechanism. 


\section{Methods}

\section{Protein expression and purification for structural studies}

MurJ $_{\mathrm{TA}}$ was expressed as described previously in 4 L culture of E. coli C41(DE3) cells transformed with a modified pET26 plasmid encoding the construct His 10 -MBP-PPX-MurJ-PPXHis $_{10}{ }^{25}$. Chloride was removed from all buffers used for cell lysis and protein purification, as detailed below. Cell pellets from each liter of culture were resuspended in $10 \mathrm{~mL}$ lysis buffer (50 mM HEPES-NaOH pH 8.0, $150 \mathrm{mM} \mathrm{Na}$ acetate) containing the following additives: $10 \mathrm{mM}$ $\beta$-mercaptoethanol, $1 \mu \mathrm{g} / \mathrm{mL}$ leupeptin, $1 \mu \mathrm{g} / \mathrm{mL}$ pepstatin, $5 \mathrm{mU} / \mathrm{mL}$ aprotinin, $20 \mu \mathrm{g} / \mathrm{mL}$ deoxyribonuclease I, and $3 \mathrm{mM}$ phenylmethylsulfonyl fluoride. Cells were disrupted by a Microfluidizer, and membrane proteins were extracted by stirring with $30 \mathrm{mM}$ n-dodecyl- $\beta$-Dmaltopyranoside (DDM, Anatrace) for 1 hour at $4^{\circ} \mathrm{C}$. Upon centrifugation at 22,000 $\mathrm{g}$ for $30 \mathrm{~min}$, the supernatant was rotated with $1.5 \mathrm{~mL}$ of TALON Cobalt resin (Clontech) for $30 \mathrm{~min}$ at $4^{\circ} \mathrm{C}$ in the presence of $5 \mathrm{mM}$ imidazole. Resin was recovered by centrifugation at $800 \mathrm{~g}$ for 5 minutes and resuspended in $7.5 \mathrm{~mL}$ of wash buffer (50 mM HEPES-NaOH pH 8.0, $150 \mathrm{mM} \mathrm{Na}$ acetate, $15 \mathrm{mM}$ imidazole, $1 \mathrm{mM}$ DDM). The slurry was poured into a gravity column, and subsequently washed twice with $7.5 \mathrm{~mL}$ of the same buffer at $4^{\circ} \mathrm{C}$. Protein was eluted in $6 \mathrm{~mL}$ of wash buffer with imidazole added to $200 \mathrm{mM}$ and incubated overnight at $4^{\circ} \mathrm{C}$ with $40 \mu \mathrm{g} / \mathrm{mL}$ PreScission protease and $5 \mathrm{mM} \beta$-mercaptoethanol. PreScission protease-treated MurJ was exchanged to gel filtration buffer, containing $20 \mathrm{mM}$ HEPES-NaOH $\mathrm{pH} 8.0,150 \mathrm{mM}$ Na acetate, $2 \mathrm{mM}$ dithiothreitol, and $0.3 \mathrm{mM}$ decyl maltose neopentyl glycol (DMNG, Anatrace), by 15-fold dilution and rotation for $15 \mathrm{~min}$ at $4^{\circ} \mathrm{C}$, and subsequently purified by gel filtration on a Superdex 200 10/300 column. 


\section{Crystallization}

MurJ $_{\text {TA }}$ was crystallized by the lipidic cubic phase (LCP) method ${ }^{41}$ in Lipid II-doped mesophase as described previously but with modifications ${ }^{25}$. Purified, chloride-free MurJ $_{\mathrm{TA}}$ was concentrated to $20-25 \mathrm{mg} / \mathrm{mL}$ with a $50 \mathrm{kD}$ molecular-weight-cutoff centrifugal filter, centrifuged at $16,000 \mathrm{~g}$ for $30 \mathrm{~min}$ at $4^{\circ} \mathrm{C}$, before mixing with molten Lipid II-doped monoolein (prepared as described previously ${ }^{25}$ ) in the standard 2:3 (w/w) water:lipid ratio using a twinsyringe setup. MurJ-reconstituted LCPs were dispensed on 96-well glass sandwich plates as 130 nL drops by a Gryphon LCP robot (Art Robbins Instruments) and overlaid with $1 \mu$ of precipitant solution from crystallization screens made in-house and/or the commercial MemMeso HT-96 screen (Molecular Dimensions).

The salt composition of the precipitant solution was the key determinant of which crystal form was obtained, which was also influenced by $\mathrm{pH}$ to a lesser extent. We did not notice any prep-to-prep variation in which crystal forms were obtained from each precipitant solution. Crystals of the inward-closed structure were obtained in $1 \mathrm{M} \mathrm{NaCl}, 50 \mathrm{mM} \mathrm{Na}$ acetate- $\mathrm{HCl} \mathrm{pH}$ 4.6, 40\% PEG400. Crystals of the inward-open structure were obtained in $200 \mathrm{mM}$ ammonium sulfate, $50 \mathrm{mM}$ Tris- $\mathrm{HCl} \mathrm{pH} 8.5,20 \%$ PEG400; or in $200 \mathrm{mM}$ ammonium sulfate, $100 \mathrm{mM} \mathrm{Na}$ citrate tribasic- $\mathrm{HCl} \mathrm{pH} \mathrm{5.5,40 \%} \mathrm{PEG400.} \mathrm{Crystals} \mathrm{of} \mathrm{the} \mathrm{inward-occluded} \mathrm{structure} \mathrm{were}$ obtained in $100 \mathrm{mM} \mathrm{Na}$ citrate tribasic-HCl pH 5.0, 40\% PEG200 (MemMeso A4). Crystals of the outward structure were obtained in $1 \mathrm{M} \mathrm{NaCl}, 50 \mathrm{mM}$ HEPES-NaOH pH 7.5, 20\% PEG400; or in $100 \mathrm{mM} \mathrm{MgCl}, 100 \mathrm{mM} \mathrm{NaCl}, 100 \mathrm{mM}$ HEPES-NaOH pH 7.0, 30\% PEG500 DME (MemMeso E9); or in $100 \mathrm{mM} \mathrm{MgCl}$, $100 \mathrm{mM} \mathrm{NaCl}, 100 \mathrm{mM}$ MES-NaOH pH 6.0, 30\% PEG500 MME (MemMeso F9). Crystals grew to full size in 4 weeks and were flash-frozen in liquid nitrogen without additional cryo-protectant. 


\section{Data collection and structure determination}

We collected X-ray diffraction data at the NECAT 24-ID-C and 24-ID-E beamlines (Advanced Photon Source, Argonne National Laboratory) with a wavelength of $\sim 0.98 \AA$. For the inward-occluded structure, we attempted to collect redundant data at a longer wavelength $(1.65 \AA)$ to locate anomalous difference Fourier electron density peaks corresponding to the diphosphate moiety of Lipid II, but without success likely due to the inherent flexibility of Lipid II ( $\sim 68$ rotatable torsions). Nevertheless, the long-wavelength datasets were merged with the datasets collected at $0.98 \AA$ for refinement as they were isomorphous and there was no noticeable radiation damage. Data were processed by XDS ${ }^{42}$, and XDS-processed data from multiple isomorphous crystals were merged by POINTLESS and AIMLESS in BLEND ${ }^{43-45}$. Due to diffraction anisotropy in the high resolution shells, merged data were subjected to anisotropy correction in STARANISO ${ }^{46}$. Anisotropy corrected data were used for molecular replacement using our previously published structure of $\operatorname{MurJ}_{\mathrm{TA}}$ (PDB: 5T77) as the search model in PHASER ${ }^{47}$. For the outward structure, the search model was split into two fragments (residues 4-228 and residues 229-470) and a two-component search yielded a strong MR solution with loglikelihood gain of 1588 and translation function Z-score of 40. MR solutions were subjected to iterative rounds of model building in COOT ${ }^{48}$ and refinement in PHENIX.refine ${ }^{49}$. Structures were refined to a final $R_{\text {work }} / R_{\text {free }}$ of $25.5 / 28.0 \%$ (inward-closed structure, $3.2 \AA$ resolution), 25.4/27.8\% (inward-open structure, $3.0 \AA$ resolution), 22.9/25.8\% (inward-occluded structure, $2.6 \AA$ resolution), and 17.9/19.9\% (outward structure, $1.8 \AA$ resolution). The final models were refined to excellent geometry ( $>97 \%$ Ramachandran favored, 0\% Ramachandran outliers, $<1 \%$ rotamer outliers) and minimal clashes (clashscore $<2.5$ ). Crystals contained either a single MurJ 
molecule in the asymmetric unit (inward-occluded and outward structures), or two MurJ molecules (inward-closed and inward-open structures).

Omit maps for $\mathrm{Na}^{+}$were calculated in PHENIX.maps to the maximum resolution of the respective datasets. For the outward-facing structure, the Fo - Fc omit peak was $19 \sigma$ for the merged data used for structure refinement, or $17 \sigma$ for the single dataset crystallized in $1 \mathrm{M} \mathrm{NaCl}$ without $\mathrm{Mg}^{2+}$, with both maps calculated to $1.8 \AA$ resolution. The Fo $-\mathrm{Fc}$ omit peak was $9 \sigma$ for the inward-occluded structure, and $7 \sigma$ for the inward-closed structure. $\mathrm{Na}^{+}$coordination geometry was validated by the CheckMyMetal server ${ }^{35}$. Structural alignments and molecular graphics were created in PyMOL ${ }^{50}$.

\section{Docking of Lipid II and molecular dynamics simulation}

The L-lysine form of Lipid II (1875 Da) was docked into the crystal structure of the inward-occluded conformation as described previously ${ }^{25}$. Briefly, Lipid II coordinates (including all hydrogens, protonation state set to neutral $\mathrm{pH}$, i.e. protonated L-lysine but deprotonated phosphates/carboxylates) were generated from 2D geometry in PHENIX.eLBOW. Stereochemistry was rigorously checked and manually corrected in PHENIX.REEL, including configuration of all 16 chiral centers, planarity and cis/trans configuration of peptide and polyprenyl $\mathrm{C}=\mathrm{C}$ double bonds, sugar pucker and glycosidic linkage. The remaining 68 torsions were set to be freely rotatable. The unresolved sidechains of Arg18 and Lys53 were built manually with the least-clashing ideal rotamer and subsequently treated as flexible for docking. Protein and ligand were prepared for docking in Autodock Tools, merging charges and removing 
non-polar hydrogens ${ }^{51}$. Docking was performed by Autodock Vina with a search space of $24 \mathrm{x}$ $24 \times 38 \AA \AA$ encompassing the central cavity, portal, and groove ${ }^{52}$.

Molecular dynamics simulations starting from the docked model were set up as follows. The protein molecule from docking was processed by PDB2PQR to add all hydrogens (not just polar hydrogens, protonation state set to neutral $\mathrm{pH}$ ) and all missing sidechains (not just those in the cavity) ${ }^{53,54}$. The protein molecule was embedded into a 1-Palmitoyl-2-oleoyl-sn-glycero-3phosphoethanolamine (POPE) membrane with xy-dimensions of 140 x $120 \AA$ with the membrane plugin (www.ks.uiuc.edu/Research/vmd/plugins/membrane) in VMD, which also removes any POPE molecules that overlap with the embedded protein ${ }^{55}$. This was performed using the CHARMM all-hydrogen forcefield topology file for protein and lipids (top_all27_prot_lipid.inp) ${ }^{56-58}$.

The Lipid II molecule in the docked conformation was processed by PHENIX.ReadySet (using the original restraints cif file from PHENIX.REEL) to add back all the hydrogens with protonation state set to neutral $\mathrm{pH}$, and the Lipid II CHARMM forcefield topology file was generated by the SWISS-PARAM server (www.swissparam.ch) ${ }^{59}$. Lipid II was then embedded into the membrane by superposing the docked protein-Lipid II complex on to the embedded protein molecule in the membrane. The embedded protein molecule (with Arg18 and Lys53 in the docked conformation), Lipid II molecule (docked conformation), and POPE lipid bilayer were combined into a single file and solvated with TIP3P waters on both sides of the bilayer to an overall z-dimension of $100 \AA$ using the Solvate plugin in VMD ${ }^{55}$. Total charge of the system was neutralized with addition of $150 \mathrm{mM} \mathrm{NaCl}$ by the Autoionize plugin in VMD ${ }^{55}$. Waters that are in the hydrophobic membrane interior (excluding those in the solvent-accessible cavity of MurJ) were manually deleted in PyMOL. There were no ions in the hydrophobic membrane 
interior. This solvated and ionized system ( 140,600 atoms) was then processed by the AutoPSF plugin in VMD to generate the combined psf and pdb files for MD simulation, using the abovementioned CHARMM forcefield topology files (top_all27_prot_lipid.inp for protein/POPE and the SWISS-PARAM output for Lipid II) ${ }^{55}$.

Molecular dynamics simulations were performed by NAMD ${ }^{60}$, with reference to a tutorial manual (www.ks.uiuc.edu/Training/Tutorials/science/membrane/mem-tutorial.pdf). For all simulation runs, an integration time step of $1 \mathrm{fs}$ was used and all $\mathrm{X}-\mathrm{H}$ bonds were set to rigid (both bond lengths and angles). A periodic cell of 140 x 120 x $100 \AA$ was used with Particle Mesh Ewald electrostatics evaluation ${ }^{61}$, wrapping both waters and POPE lipid molecules that diffuse out of the boundaries back into the opposite side of the cell. To mimic the disorder in an actual membrane, the lipid tails of POPE molecules were first melted while everything else (including POPE headgroups) were fixed in position. Subsequently, gaps between the protein and POPE molecules were filled by a constrained equilibration run at $310 \mathrm{~K}$ and $1 \mathrm{~atm}$, with energy minimization for 1000 steps and simulation for $100 \mathrm{ps.} \mathrm{The} \mathrm{follower} \mathrm{parameters} \mathrm{were}$ also used: flexible cell, constant ratio, fixed atoms released, harmonic constraints on protein, keep_waters_out.tcl script to keep waters out from the bilayer). Finally, a production run was performed at $310 \mathrm{~K}$ and 1 atm for $100 \mathrm{ps}$ releasing the constraints, and coordinates were recorded at $1 \mathrm{ps}$ intervals. At the end of simulation, the stereochemistry of Lipid II was checked and no distortion was found.

\section{Expression and delipidation of single cysteine mutants for in vitro assay}


Because MurJ $_{\mathrm{TA}}$ has no endogenous cysteine residues, single cysteine mutants could be engineered at either the cytoplasmic and/or periplasmic gates to probe the protein conformation in vitro in the absence or presence of sodium. One liter of each cysteine mutant was expressed as described for the structural studies. Sodium was removed from all cell lysis and protein purification buffers, replaced by either N-methyl-D-glucamine (NMDG) or potassium. Chloride was replaced by acetate in all buffers. The protein purification procedure in NMDG is detailed below. Cell pellets from each liter of culture were resuspended in $10 \mathrm{~mL}$ lysis buffer, which contained $50 \mathrm{mM}$ Tris-acetate and $150 \mathrm{mM}$ NMDG, $\mathrm{pH}$. This buffer was made by dissolving the free base of both Tris and NMDG together, then adjusting to $\mathrm{pH} 8$ with glacial acetic acid. The same additives were used in the lysis buffer as for structural studies, with the sole exception of $\beta$-mercaptoethanol being replaced by $1 \mathrm{mM}$ Tris(2-carboxyethyl)phosphine (TCEP, Thermo Fisher). Cell disruption, membrane extraction, and cell debris removal were performed as described for structural studies. The supernatant was rotated with $0.5 \mathrm{~mL}$ of TALON Cobalt resin (Clontech) for $30 \mathrm{~min}$ at $4^{\circ} \mathrm{C}$ in the presence of $5 \mathrm{mM}$ imidazole. Resin was recovered by centrifugation at $800 \mathrm{~g}$ for 5 minutes and resuspended in $5 \mathrm{~mL}$ of wash buffer $(50 \mathrm{mM}$ Trisacetate and $150 \mathrm{mM}$ NMDG, $\mathrm{pH} 8,15 \mathrm{mM}$ imidazole, $1 \mathrm{mM}$ TCEP, $1 \mathrm{mM}$ DDM). The slurry was poured into a gravity column, and subsequently washed twice with $5 \mathrm{~mL}$ of the same buffer at $4^{\circ} \mathrm{C}$. Protein was eluted in $4 \mathrm{~mL}$ of wash buffer with imidazole added to $200 \mathrm{mM}$, then incubated overnight at $4^{\circ} \mathrm{C}$ with $20 \mu \mathrm{g} / \mathrm{mL}$ PreScission protease without $\beta$-mercaptoethanol.

As the presence of any cardiolipin that is carried over from E. coli lysate could be inhibitory to MurJ function ${ }^{20}$, we took measures to delipidate our MurJ samples. We exploited the high isoelectric point of $\operatorname{MurJ}_{\mathrm{TA}}$ (predicted to be 8.5-9.5) to delipidate MurJ $_{\mathrm{TA}}$ by cation exchange. PreScission protease-treated proteins were diluted fivefold in buffer A (20 mM Tris- 
acetate $\mathrm{pH}$ 8.0, $1 \mathrm{mM}$ DDM), treated with $1 \mathrm{mM}$ TCEP, and passed through $0.5 \mathrm{~mL}$ of preequilibrated sulfopropyl(SP)-sepharose fast-flow resin (GE healthcare) twice in a gravity column at room temperature. The resin was washed with $5 \mathrm{~mL}$ each of $50 \mathrm{mM}$ NMDG (16.7\% buffer B), $100 \mathrm{mM}$ NMDG (33.3\% buffer B), and $150 \mathrm{mM}$ NMDG (50\% buffer B), before elution in $2 \mathrm{~mL}$ of $300 \mathrm{mM}$ NMDG (100\% buffer B, divided into four $0.5 \mathrm{~mL}$ fractions), all performed at room temperature. Buffer B contained 50 mM Tris-acetate pH 8.0, 300 mM NMDG, 1 mM DDM). Eluted protein was extremely pure (single band on SDS-PAGE) and mass spectrometry analysis indicated that all traces of cardiolipin had been successfully removed by the delipidation treatment. We did not perform delipidation for structural studies because the high purity came at the cost of drastically reduced recovery $(10-30 \mathrm{ug} / \mathrm{L}$ culture). Eluted protein was concentrated to 3-5 uM with a $50 \mathrm{kD}$ MW cutoff centrifugal filter before the assay.

\section{In vitro cysteine accessibility assay (direct labeling)}

To probe the conformation of MurJ $_{\mathrm{TA}}$ in vitro, single-cysteine mutants (no endogenous cysteines) were purified and delipidated in potassium acetate buffer as detailed above. Mutants were diluted to $\sim 1 \mathrm{uM}$ in reaction buffer containing $20 \mathrm{mM}$ HEPES-KOH pH 7.5, $1 \mathrm{mM}$ DDM, and $150 \mathrm{mM}$ potassium acetate (buffer was added from $5 \mathrm{x}$ stock). Samples $(9 \mathrm{uL})$ were equilibrated at either $20^{\circ} \mathrm{C}$ or $60^{\circ} \mathrm{C}$ for 10 minutes, then treated with $1 \mathrm{uL}$ of methoxypolyethylene glycol 5000 maleimide (PEG-Mal, Sigma-Aldrich, 10x stock in water) to a working concentration of $0.5 \mathrm{mM}$. An equivalent volume of water was added to the negative control samples. Reactions were allowed to proceed at either $20^{\circ} \mathrm{C}$ or $60^{\circ} \mathrm{C}$ for 10 minutes, and subsequently quenched with $5 \mathrm{mM}$ N-ethyl-maleimide (NEM, Sigma-Aldrich, 10x stock 
dissolved in ethanol) for 10 minutes at room temperature. Samples were mixed with $2 \mathrm{x}$ denaturing buffer (60 mM Tris- $\mathrm{HCl} \mathrm{pH}$ 6.8, $8 \mathrm{M}$ urea, 4\% (w/v) SDS, 20\% (v/v) glycerol, and $0.01 \%(\mathrm{w} / \mathrm{v})$ bromophenol blue) in a $1: 1$ volume ratio, resolved by SDS-PAGE on a $10 \%$ polyacrylamide gel, and stained by Coomassie brilliant blue R-250.

\section{In vitro cysteine accessibility assay (inverse labeling)}

To probe changes in accessibility in the presence of sodium, single-cysteine mutants were purified and delipidated in NMDG buffer as detailed above. Samples were diluted to $\sim 1 \mathrm{uM}$ in reaction buffer containing $20 \mathrm{mM}$ Tris-acetate $\mathrm{pH}$ 7.5, $1 \mathrm{mM}$ DDM, and either $150 \mathrm{mM}$ sodium acetate or $150 \mathrm{mM}$ NMDG (buffers were added from $5 \mathrm{x}$ stocks). Samples (9 uL) were equilibrated at $20^{\circ} \mathrm{C}$ for 10 minutes, then treated with 1 uL of sodium 2-sulfonatoethyl methanethiosulfonate (MTSES, Anatrace, 10x stock in water) to a working concentration of 100 uM. An equivalent volume of water was added to the negative control samples. Because long MTSES incubation times were found to reduce sensitivity by excessively blocking residues that should be inaccessible, $10 \mathrm{uL}$ of $2 \mathrm{x}$ denaturing/labeling buffer was immediately added for a MTSES blocking time of approximately 2 minutes. This $2 \mathrm{x}$ denaturing/labeling buffer contained $50 \mathrm{mg} / \mathrm{mL}(\sim 10 \mathrm{mM})$ methoxypolyethylene glycol 5000 maleimide (PEG-Mal, Sigma-Aldrich) dissolved in the $2 \mathrm{x}$ denaturing buffer described above. Samples were immediately homogenized by brief vortex and spun down. Denaturation and labeling proceeded for 30 minutes under protection from light. Reactions were quenched by addition of $1 \%(\mathrm{v} / \mathrm{v}) \beta$-mercaptoethanol (from 10\% stock). Samples were resolved by SDS-PAGE and stained as described above. Gel band intensities were quantified in GelQuant.NET and fold change was determined by dividing 
the relative accessibility in sodium by the relative accessibility in NMDG. Relative accessibilities were calculated as follows:

$$
\text { Relative accessibility }=\frac{\text { Bottom band (MTSES) }}{\text { Top band (MTSES) }}-\frac{\text { Bottom band (no MTSES) }}{\text { Top band (no MTSES) }}
$$

\section{In vivo complementation assay}

MurJ $_{\mathrm{TA}}$ mutants was generated by site-directed mutagenesis using standard recombinant DNA methods from MurJ $\mathrm{TA}_{\mathrm{TA}}$ wild-type in $\mathrm{pEXT} 21$ vector ${ }^{25}$. The plasmid carrying MurJ $_{\mathrm{TA}}$ (wildtype or mutants) were transformed into MurJ-depletion E.coli strain NR1154 ${ }^{8}$. Growth of NR1154 is dependent on L-arabinose, while treatment with the anti-inducer D-fucose depletes endogenous MurJ. Transformants were selected with $80 \mu \mathrm{g} / \mathrm{ml}$ spectinomycin on LB-agar plates supplemented with $0.2 \%$ of arabinose. Overnight cultures were diluted 1:1000 into fresh LB media with $80 \mu \mathrm{g} / \mathrm{ml}$ spectinomycin and $0.2 \%$ of arabinose were grown to $\mathrm{OD}_{600}$ of 0.6 . Cultures were then split into two halves and a final concentration of $0.1 \mathrm{mM}$ IPTG was added to one half. Cultures were further incubated for $3 \mathrm{~h}$ at $37^{\circ} \mathrm{C}$. Cells were collected by centrifugation and washed with fresh LB three times and normalized to an $\mathrm{OD}_{600}$ of 0.2. Each culture was then diluted to 0.02 , subjected to ten-fold serial dilution, and $5 \mu 1$ were spotted onto LB-agar plates supplemented with either $0.05 \%$ fucose or $0.05 \%$ of fucose with $0.1 \mathrm{mM}$ IPTG. To determine whether mutants were expressed, total membrane fractions were isolated and analyzed by western blot as was described previously ${ }^{25}$; primary antibody: mouse anti-FLAG (Sigma F3165); secondary antibody: horseradish peroxidase-conjugated goat anti-mouse (Invitrogen \#31430). 


\section{In vivo Lipid II accumulation assay}

The plasmid carrying MurJ $_{\mathrm{TA}}$ (wild-type, mutant, or empty vector) were transformed into E. coli NR1154 and transformants were selected with $80 \mu \mathrm{g} / \mathrm{ml}$ spectinomycin on LB-agar plates supplemented with $0.2 \%$ of arabinose. Overnight cultures were normalized to an $\mathrm{OD}_{600}$ of 1.5 and diluted 1:10,000 into fresh LB media supplemented with $80 \mu \mathrm{g} / \mathrm{ml}$ spectinomycin and $0.05 \%$ of fucose. $\mathrm{OD}_{600}$ were measured every hour after $2 \mathrm{~h}$ of shaking at $37{ }^{\circ} \mathrm{C}$. After 6 hours, cells were normalized to the lowest $\mathrm{OD}_{600}$ of all samples and collected by centrifugation for mass spectrometry analysis.

Lipid extraction was performed using a modified Bligh-Dyer method ${ }^{62}$ Specifically, each cell pellet was re-suspended in $0.8 \mathrm{~mL}$ of $1 \times$ PBS (phosphate-buffered saline), and transferred into a $17 \mathrm{~mL}$ glass tube with a Teflon-lined cap, which is then followed by the addition of $2 \mathrm{~mL}$ of methanol and $1 \mathrm{~mL}$ of chloroform to create a single-phase Bligh-Dyer solution (chloroform/methanol/PBS, 1:2:0.8, v/v/v). The solution was vigorously vortexed for $2 \mathrm{~min}$, followed by sonication in a water bath at room temperature for $20 \mathrm{~min}$. After centrifugation at $3,000 \times \mathrm{g}$ for $10 \mathrm{~min}$, the supernatant was transferred to a fresh glass tube and acidified by adding $50 \mu \mathrm{L}$ of concentrated $\mathrm{HCl}(37 \%)$. After mixing, the acidified solution was converted into a twophase Bligh-Dyer system by adding $1 \mathrm{~mL}$ of PBS and $1 \mathrm{~mL}$ of chloroform. After centrifugation at 3,000 $\times \mathrm{g}$ for $10 \mathrm{~min}$, the lower phase was collected and dried under nitrogen gas. For Lipid II and Lipid X analysis, the dried lipid extracts were dissolved in a $100 \mu$ l solution consisting of chloroform/methanol (2:1, v/v), of which $20 \mu$ was injected for normal phase LC/MS analysis.

Normal phase LC was performed on an Agilent 1200 Quaternary LC system equipped with an Ascentis Silica HPLC column (5 $\mu \mathrm{m}, 25 \mathrm{~cm}$ x $2.1 \mathrm{~mm}$, Sigma-Aldrich, St. Louis, MO). 
Mobile phase A consisted of chloroform/methanol/aqueous ammonium hydroxide (800:195:5, $\mathrm{v} / \mathrm{v} / \mathrm{v})$; mobile phase B consisted of chloroform/methanol/water/aqueous ammonium hydroxide $(600: 340: 50: 5, \mathrm{v} / \mathrm{v} / \mathrm{v})$; mobile phase $\mathrm{C}$ consisted of chloroform/methanol/water/aqueous ammonium hydroxide (450:450:95:5, v/v/v). The elution program consisted of the following: $100 \%$ mobile phase A was held isocratically for 2 min and then linearly increased to $100 \%$ mobile phase B over $14 \mathrm{~min}$ and held at $100 \% \mathrm{~B}$ for $11 \mathrm{~min}$. The LC gradient was then changed to $100 \%$ mobile phase $\mathrm{C}$ over $3 \mathrm{~min}$ and held at $100 \% \mathrm{C}$ for $3 \mathrm{~min}$, and finally returned to $100 \%$ A over $0.5 \mathrm{~min}$ and held at $100 \% \mathrm{~A}$ for $5 \mathrm{~min}$. The LC eluent (with a total flow rate of 300 $\mu \mathrm{l} / \mathrm{min}$ ) was introduced into the ESI source of a high resolution TripleTOF5600 mass spectrometer (Sciex, Framingham, MA). Instrumental settings for negative ion ESI and MS/MS analysis of lipid species were as follows: $\mathrm{IS}=-4500 \mathrm{~V}$; $\mathrm{CUR}=20 \mathrm{psi}$; GSI= 20 psi; $\mathrm{DP}=-55 \mathrm{~V}$; and $\mathrm{FP}=-150 \mathrm{~V}$. The MS/MS analysis used nitrogen as the collision gas. Data analysis was performed using Analyst TF1.5 software (Sciex, Framingham, MA). The levels of Lipid II, Lipid $\mathrm{X}$, Phosphatidyethanolamine were quantified by using the integrated peak areas of their extracted ion chromatograms $\left(\mathrm{m} / \mathrm{z} 710.4\right.$ for the $[\mathrm{M}-\mathrm{H}]^{-}$ion of Lipid $\mathrm{X}$ and 958.4 for the $[\mathrm{M}-2 \mathrm{H}]^{2-}$ ion of Lipid II).

\section{Data and materials availability}

Atomic coordinates and structure factors for the reported crystal structures are deposited in the Protein Data Bank under accession codes 6NC6, 6NC7, 6NC8, and 6NC9. Any other data pertaining to this paper is available upon request. Correspondence and requests for materials should be addressed to S.-Y.L. (seok-yong.lee@duke.edu). 
Extended Data Table 1. Data collection and refinement statistics

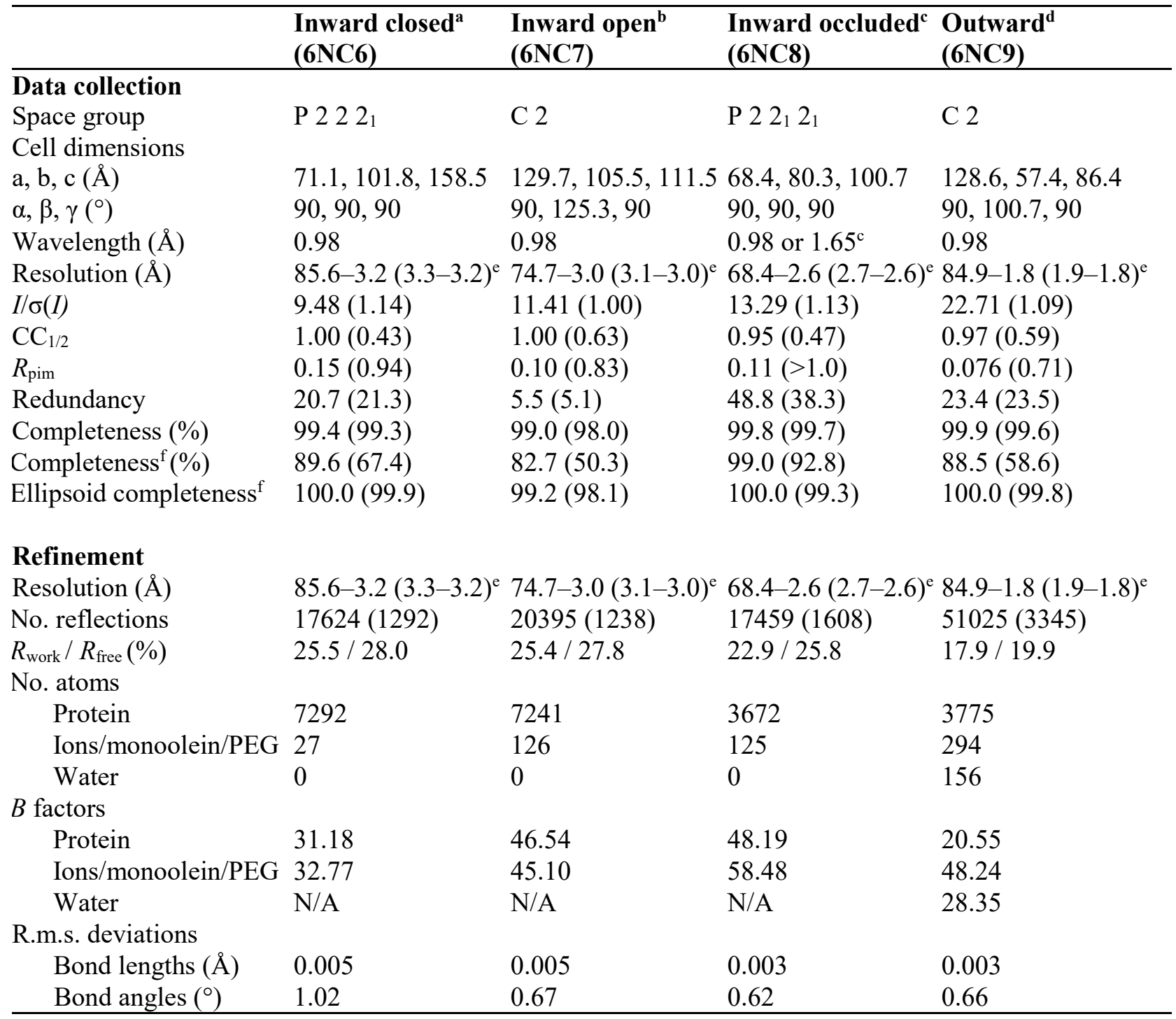

${ }^{a}$ Merged from 3 crystals.

${ }^{\mathrm{b}}$ Merged from 2 crystals.

${ }^{\mathrm{c}}$ Merged from 7 crystals ( 2 collected at wavelength of $0.98 \AA$ and 5 at $1.65 \AA$ ).

${ }^{\mathrm{d}}$ Merged from 6 crystals.

${ }^{\mathrm{e}}$ Values in parentheses are for highest-resolution shell.

${ }^{\mathrm{f}}$ After applying ellipsoidal truncation and anisotropy correction in STARANISO. Ellipsoid completeness was calculated by STARANISO. All other statistics were calculated in PHENIX. 
Extended Data Table 2. Sodium Fo - Fc omit peak heights and coordination distances

\begin{tabular}{lllllll}
\hline Structure & $\begin{array}{l}\mathbf{N a}^{+} \mathbf{F o}-\mathbf{F c} \\
\text { omit peak } \\
\text { height }\end{array}$ & \multicolumn{5}{c}{$\mathbf{N a}^{+}$coordination distance (in Ångstrom) } \\
& $\begin{array}{l}\text { Asp235 } \\
\text { OD1 }\end{array}$ & $\begin{array}{l}\text { Asn374 } \\
\text { OD1 }\end{array}$ & $\begin{array}{l}\text { Asp378 } \\
\text { OD1 }\end{array}$ & Val390 & Thr394 & OG1 \\
\hline Inward closed & 7 sigma & 2.49 & 2.32 & 2.45 & 2.39 & 2.35 \\
Inward occluded & 9 sigma & 2.44 & 2.43 & 2.40 & 2.40 & 2.41 \\
Outward & 19 sigma & 2.45 & 2.26 & 2.42 & 2.33 & 2.33 \\
\hline
\end{tabular}



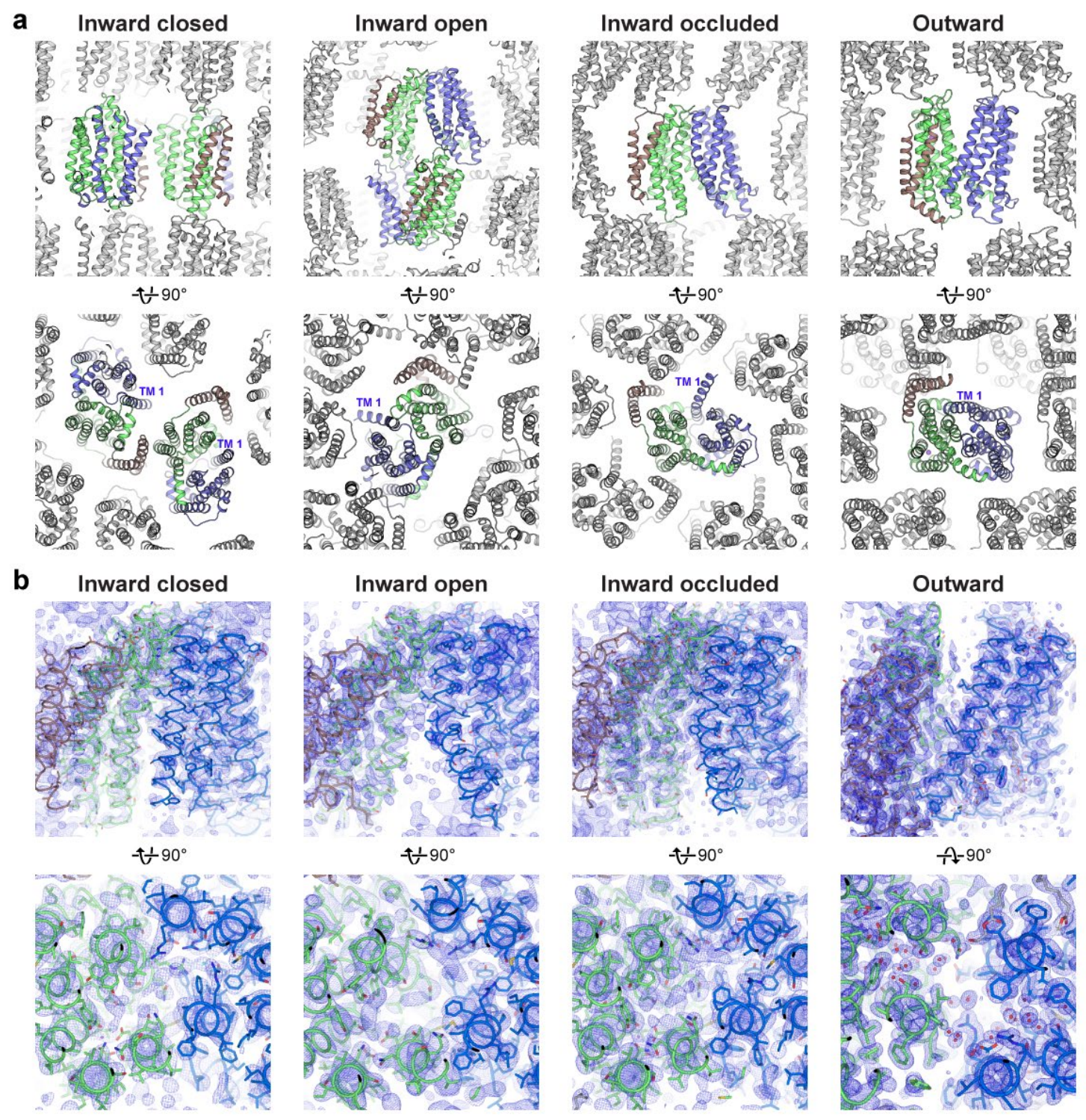

Extended Data Fig. 1 | Crystal packing and electron density in the MurJ $\mathbf{J}_{\text {TA }}$ structures. a,

Crystals were obtained in lipidic cubic phase doped with Lipid II. Crystal contacts are mostly formed by end-to-end stacking, as well as lateral interactions mediated by the host lipid monoolein. TM 1 was not involved in crystal packing and thus its bending is unlikely to be a crystallographic artifact. The asymmetric unit is colored, while symmetry mates are shown in gray. b, $2 \mathrm{Fo}-\mathrm{Fc}$ composite omit electron density maps (contoured to $1 \sigma$ ), omitting $5 \%$ of the 
bioRxiv preprint doi: https://doi.org/10.1101/552745; this version posted February 19,2019 . The copyright holder for this preprint (which was not certified by peer review) is the author/funder. All rights reserved. No reuse allowed without permission.

model at a time. Maps were calculated to resolutions of (from left) $3.2 \AA, 3.0 \AA, 2.6 \AA$, and 1.8 $\AA$. 


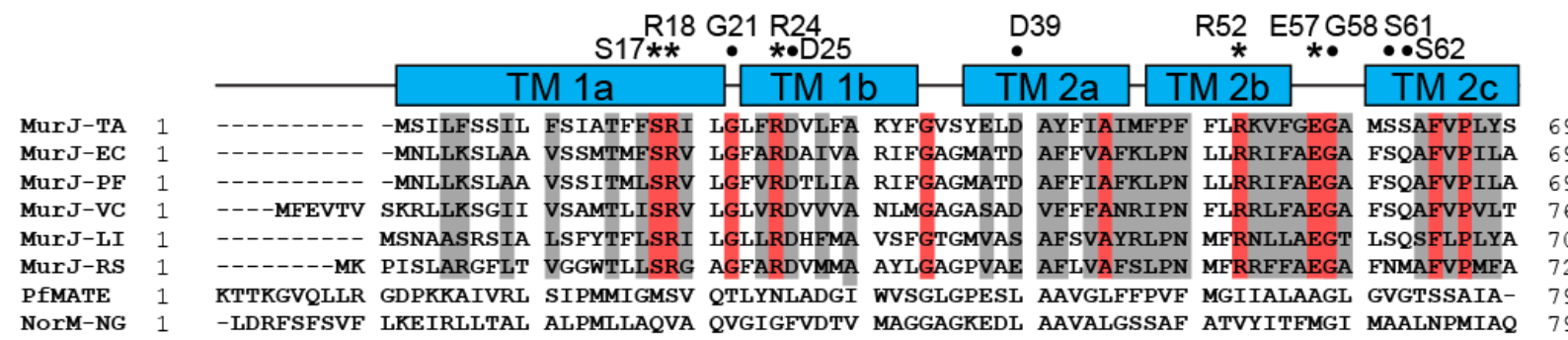

MurJ-VC 77 EYHASGDINK TRDLIARASG TLGVLVTIVT LIGVLGSGAV TALFGAGWFL DWLNGGPAAG KFELASLLLK ITFPYLWFIT MurJ-LI 71 ESGKISE-EE AKIMSGAVLS FLFFILSILV GIVFLFSPFF LPILVGG--- -------TKE YSNLVIELTY ILFFLIVTAS MurJ-RS 73 KKLEGH--ED AKAFARDAFS GLAGILVVFT LLGTLLMPWL VLAMASG--- -----FAGDA RFDLAVEFGR IAFSYIFFIS NorM-NG 80 LYGAGKT-GE AGETGRQGIW FGLILGIFGM ILMWA-AITP FRNWLTL--- -----SDYVE GTMAQYMLFT SLAMPAAMVH L145 N146 F151 P158 N162

TM 4b

MUrJ-TA 137 LWAISYSILN TNNKFFWPAL TPSISNITII IGTFLSTKY- -----GIISP TIGFLIGSIL MFFSIIKSII KHKYY----MUrJ-EC 143 LASLVGAILN TWNRFSIPAF APTLLNISMI GFALFAAPYF -NP--PVLAL AWAVTVGGVL QLVYQLPHLK KIGMLVLP-MUrJ-PF 143 LSSLAGAILN TWNRFSVPAF VPTLLNVSMI VFAVFLTPYF -DP--PVMAL GWAVLVGGLA QLLYQLPHLK KIGMLVLP-MUrJ-VC 157 FVALSGAILN TLGKFAVSSF TPVFLNVMMI LCAWYLSPNL -EQ--PEVGL AIGVFLGGLV QFLFQLPFLI KAGVLVRP-MUIJ-LI 140 LSAIFMAISN SKNRFFVPSL SPIILNLCYL FVFICLFPFV DDLHDRVIVL CFAIITGGFL QLVVQIWYVW KNKDMPKI-MurJ-RS 143 LVALLSGVLN AFGRFTEASF VPVLMNLMFI AAMLIADRQG -WD--MGLTL AWTVPVTGVA QFLFTWFAAR RLGFTLWP-PfMATE 147 FNNVGNGILR GEGDANRAML AMVLGSGLNI VLDPIFIYTL -GF--GVVGA AYATLLSMVV TSLFIAYWLF VKRDTYVDIT NOIM-NG 150 RALHAYASSL -NRPRLIMLV SFAAFVLNVP LNYIFVYGKF GMPALGGAGC GVATMAVFWF SALALWIYIA KEKFFRPFGL

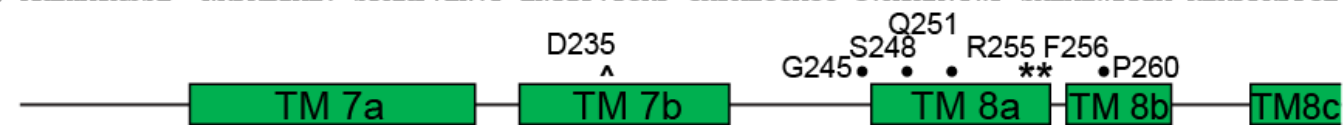

MurJ-TA 205 ------FTIK HFPHFLKLFF PTFMTMVVSO INTVVDMNVV SFYD-----K GSISYLQYAS RFYLLPYGLF AVSVSTVVLS MurJ-EC 217 -RIN--FHDA GAMRVVKQMG PAILGVSVSQ ISLIINTIFA SFLA-----S GSVSWMYYAD RLMEFPSGVL GVALGTILLP MUrJ-PF 217 -RLN--LRDS GVWRVMKQMI PAILGVSVSQ ISLIINTIFA SFLV-----A GSVSWMYYAD RLMELPSGVL GVALGTILLP MurJ-VC 231 -KWG--WKDP GVVKIRTLMI PALFGVSVSQ INLLFDSFVA SFLQ-----T GSISWLYYSD RLLEFPLGLF GIAIATVILP MurJ-LI 217 -NWN--WKHP SIRKIFKLMI PAALGGGFYQ LSLLVDIFLA NWVQNQNPGL GAVVSLDYSQ RLVQLPTGII GVALATTILP MurJ-RS 217 -HLP--RLTP DLKRLAVIAA PAVLAGGVVQ VNLLVGRQVA SFT-----E GAVAWLSYAD RLYQLPLGVV GIAIGTVLLP PfMATE 224 LRDFSPSREI LKDILRVGLP SSLSQLSMSI AMFFLNSVAI TAGG-----E NGVAVFTSAW RITMLGI-VP ILGMAAATTS NOrM-NG 229 TAKFGKPDWA VFKOIWKIGA PIGLSYFLEA SAFSFIVFLI APFG-----E DYVAAOQVGI SLSGILY-MI POSVGSAGTV - G340 $\bullet P 300$ TM 9a

MUrJ-TA 275 KISNDRKN-- ---FNYHLND ALKTTLFFTI PSMVGLIFLS TPIIRFFYEH GAFTSKDTLI TSKILIAYTL GLPFYGIYST MUIJ-EC 290 SLSKSFASGN HDEYNRLMDW GLRLCFLLAL PSAVALGILS GPLTVSLFQY GKFTAFDALM TQRALIAYSV GLIGLIVVKV MurJ-PF 290 TLAKTYASQD RHEYSRILDW GLRLCFVLVL PCALALGILA EPLTVSLFQY GQFNAFDALM TQRALIAYSV GLLGIIVIKV MurJ-VC 304 ALSRKHVDAH SDGFAHTMDW GIRMVTFLGI PAMLGLMVLA KPMLMVLFMR GEFTPSDVEQ ASYSLLAYSS GLLSFMLIKV MurJ-LI 295 ALLQSLKKEE WSSIHQELAG ALEFALFLTV PAALGMTFLA GPILDSIYFG GKWDHIATHT ATQPLVFYSI AIPFFSINKI MurJ-RS 289 DLSRRLRAGD EAGSRASFNR GTEFALMLTV PAAVALVVIA LPLTQVLFQR GAFGPEDAWN TALALGAYGL GLPAFVLHKV PAMATE 298 VTGAAYGERN VEKLETAYLY AIKIAFMIEL AVVAFIMLFA PQVAYLFTAQ VI-----KGD LISALRTLPV FLVLTPFGMM NOIM-NG 303 RIGFSLGRRE FSRARYISGV SLVSGWVLAV ITVLSLVLFR SPLASMYNDD PAVLSI---- ASTVLLFAGL FQPADFTQCI (K368 in MurJ-EC)

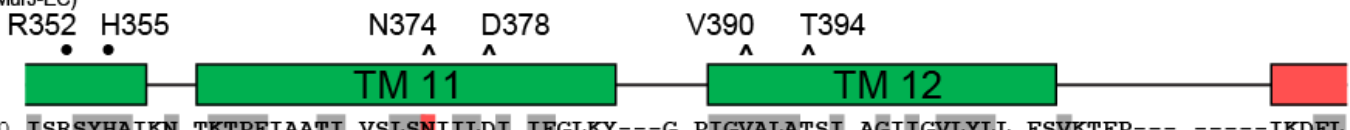

MUrJ-TA 350 ISRSYHAIKN TKTPFIAATI VSLSNIILDI IFGLKY---G PIGVALATSI AGIIGVLYLL FSVKTFP--- -----IKDFL MUIJ-EC 370 LAPGFYSRQD IKTPVKIAIV TLILTQLMNL AFIGPL---K HAGLSLSIGL AACLNASLLY WQLRKQKIFT PQPGWMAFLL MurJ-PF 370 LAPGFYAQQN IRTPVKIAIF TLVMTQLFNL VLIGPL---A HAGLALAISA GACLNAGLLF YQLRKQKMYQ PQPGWGKFGL MurJ-VC 384 LAPGYYSRQD TKTPVRYGII AMVSNIVLNA IFAWFY---G YVGLAVATSM SAFLNMALLY RGLHLQGVYH LTRKTVWFVA MUrJ-LI 375 LISSYYAFQD TKTPLRIQSI SFTINIILNL SLIWFL---K HSAIALSSAI SAIFTFLLLG IFLKKHKIGF PWFELFKKIS MurJ-RS 369 LQPLYYARED TRRPFHYAVV SMVVNLAFAI GLMPVM---G FLAAALATTV SGWVMVWQLW AGSRGMGAAS RLDERLRARL PfMATE 373 TSAMFQGIGE GEKSLILTIF RTLVMQVGFA YIFVHYTTLG LRGVWIGIVI GNMVAAIVGF LWGRMRISAL KKT------NOIM-NG 379 ASYALRGYKV TKVPMFIHAA AFWGCGLLPG YLLAYRFDMG IYGFWTALIA SLTIAAVALV WCLEKYSMEL VKSHKAVSSG

MUIJ-TA 419 KISLNSLIML FVIYLTDFT- -------- ------DNE FWFLIQILIG ILVYLIFSSI FYRDLIRRFL YARKK----MurJ-EC 447 RLVVAVLVMS GVLLGMLHIM PEWSLG---- ----TMPWRL LRLMAVVLAG IAAYFAALAV LGFKVKEFAR RTV------MurJ-PF 447 KLLVAVAVMS AVLLGAMHFM PAWGEG---- ----QMLERF LRLGALVVAG VVAYFGMLLL MGFRLRDFNR KALS-----MurJ-VC 461 RLAMAGAVMT GALLWQLDTM ATWLSW---- ----GISQRA LTLTGLIGLG VASYLAILLL LGVRLKDLKA ATE------MUrJ-LI 452 KMSIPFLLLG SYLFFHQFFL YSTILNYLGS IGVNYVQSSR IHLSLVIFPA VLIFFASSLI FKVDGIYLLI RKFRRKQEIV MurJ-RS 446 PRIVVAALFM GLVLFGATGL LEPLLSE--- -----PGWRY PGLAALVAAG IASYFGAGAA IGAFRLSDLR SLRRRA----

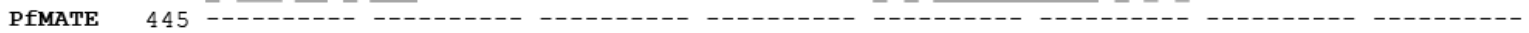
NorM-NG 
Extended Data Fig. 2 | Alignment of MurJ sequences. Positions shaded in red are identical in $>90 \%$ of Gram-negative MurJ sequences in an alignment with 36 sequences, while those shaded gray are similar in $>70 \%$ of the sequences. Asterisks (*) denote essential residues in MurJ $_{\text {TA }}$ of which alanine mutants fail to complement E. coli MurJ, as determined previously ${ }^{25}$. Carets $\left({ }^{\wedge}\right)$ denote residues participating in $\mathrm{Na}^{+}$coordination. Bullets $(\bullet)$ denote other residues discussed in this manuscript. MurJ $\mathrm{T}_{\mathrm{TA}}$ from Thermosipho africanus (UniProt ID: B7IE18) was aligned with 36 Gram-negative MurJ sequences from the UniRef50 library (no sequence is more than $50 \%$ identical to another). Drug exporters PfMATE and NorM-NG were also included in the alignment for contrast.

List of sequences shown: MurJ-TA, Thermosipho africanus (UniProt ID: B7IE18); MurJ-EC, Escherichia coli (P0AF16); MurJ-PF, Pseudomonas fluorescens (G8PWV6); MurJ-VC, Vibrio cholerae (O34238); MurJ-LI, Leptospira interrogans (A0A0C5X9A5); MurJ-RS, Rhodobacter sphaeroides (Q3J5H7); PfMATE (PDB ID: 3VVN); NorM-NG (4HUK). 

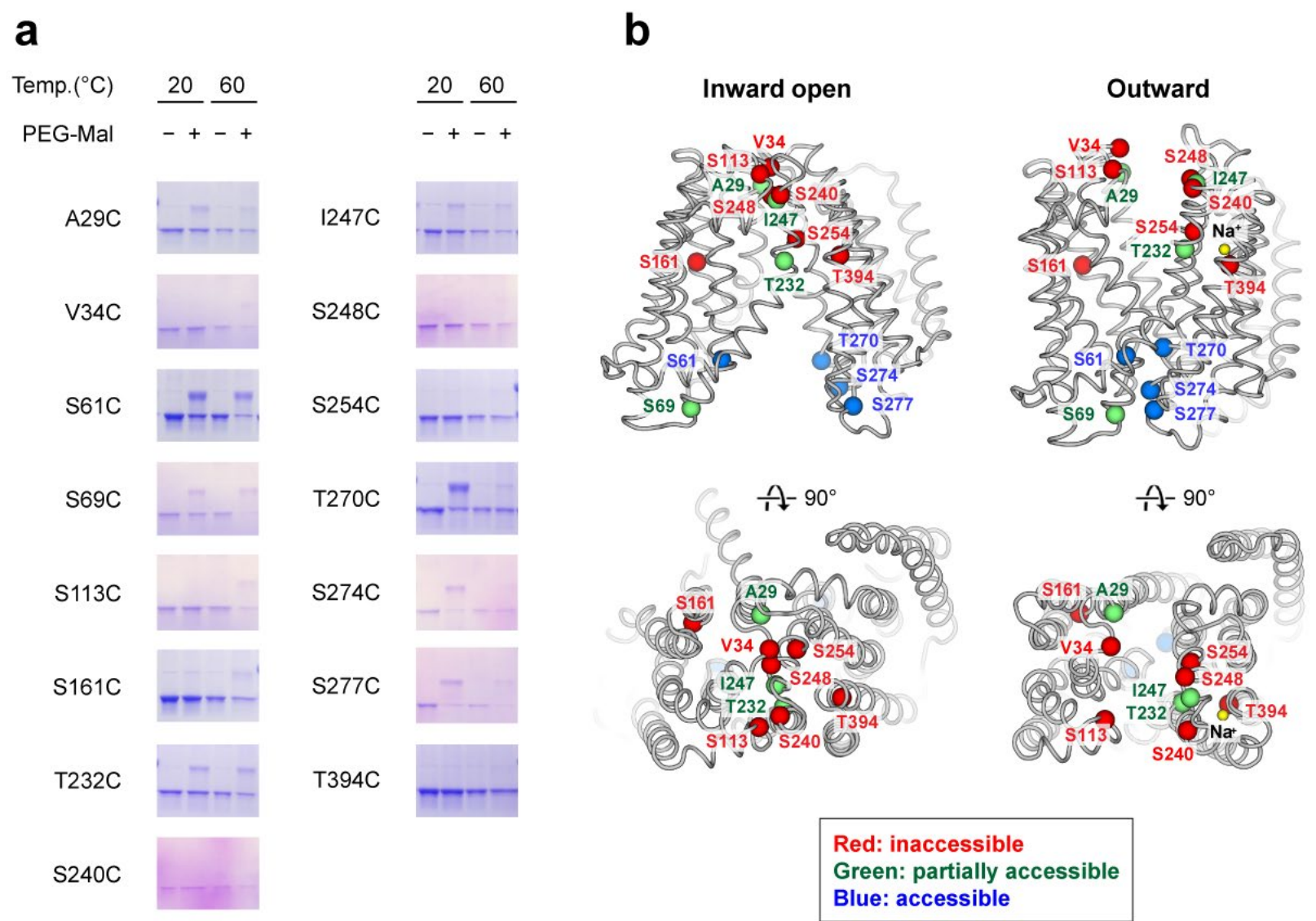

\section{Extended Data Fig. 3 | MurJ $\mathbf{J}_{\mathrm{TA}}$ assumes a predominantly inward-facing conformation in}

DDM micelles. a, Single cysteine mutants of $\mathrm{MurJ}_{\mathrm{TA}}$ (no endogenous cysteines) were expressed, purified, delipidated, and probed for accessibility in DDM micelles by a direct labeling strategy in potassium acetate (absence of sodium). Accessible cysteines would be labeled by PEG5000maleimide (PEG-Mal), resulting in a mass shift up the gel. Experiments were performed at either $20^{\circ} \mathrm{C}$ or $60^{\circ} \mathrm{C}$. b, Mapping of these cysteine positions (for the $20^{\circ} \mathrm{C}$ data) to the inwardopen and outward-facing crystal structures of MurJ $\mathrm{T}_{\mathrm{TA}}$. Positions were considered inaccessible if only the lower band was observed, and accessible if the top band was observed and of higher intensity than the lower band. Remaining positions with both bands visible were considered partially accessible. 


\section{a}

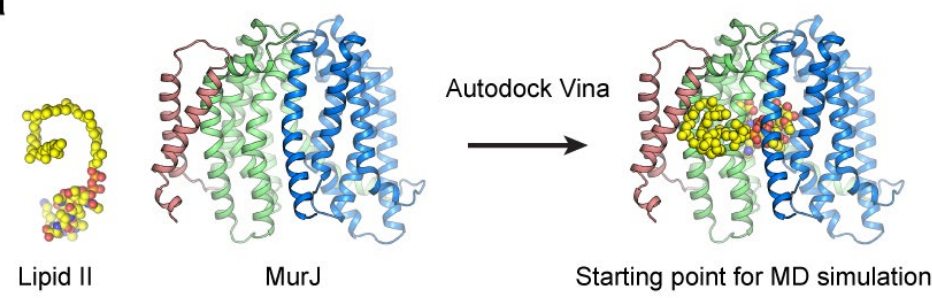

b

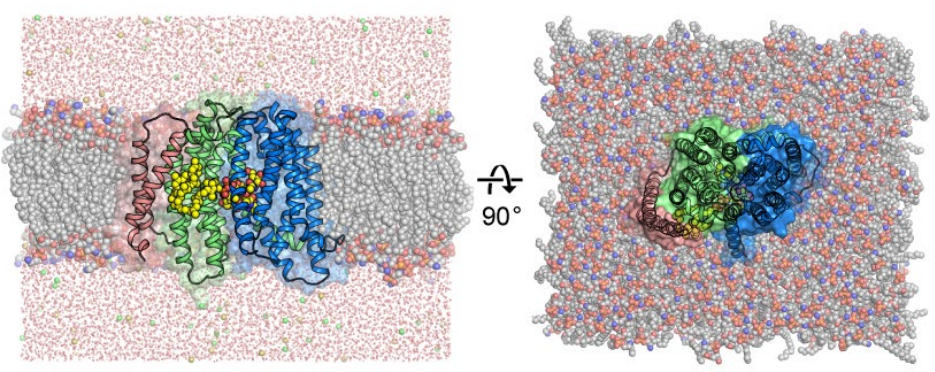

c

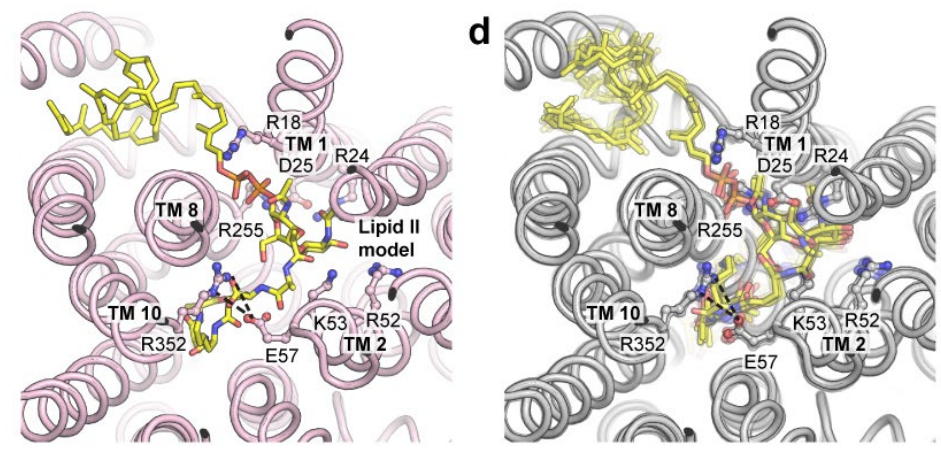

Extended Data Fig. 4 | Docking and MD simulation of Lipid II to the inward-occluded

structure. a, Lipid II (yellow spheres) was docked to the inward-occluded structure by Autodock Vina. b, The docking result was used as a starting point for molecular dynamics simulation by NAMD in a hydrated and ionized $(150 \mathrm{mM} \mathrm{NaCl})$ POPE membrane bilayer system. The equilibrated system is shown, with lipids visualized as spheres and waters denoted by red dots. c, Cytoplasmic view of the docked complex (Lipid II shown as yellow sticks, charged residues in the cavity shown as gray sticks), showing how the Glu57-Arg352 thin gate could help occlude the substrate. d, Rotation of MurJ sidechain and Lipid II torsions in a molecular dynamics simulation starting from the docked model, visualizing the picosecond 

not certified by peer review) is the author/funder. All rights reserved. No reuse allowed without permission.

dynamics of the docked MurJ-Lipid II complex (snapshots at each 10 ps overlaid, total 100 ps).

Surrounding lipids and waters are hidden for clarity. 


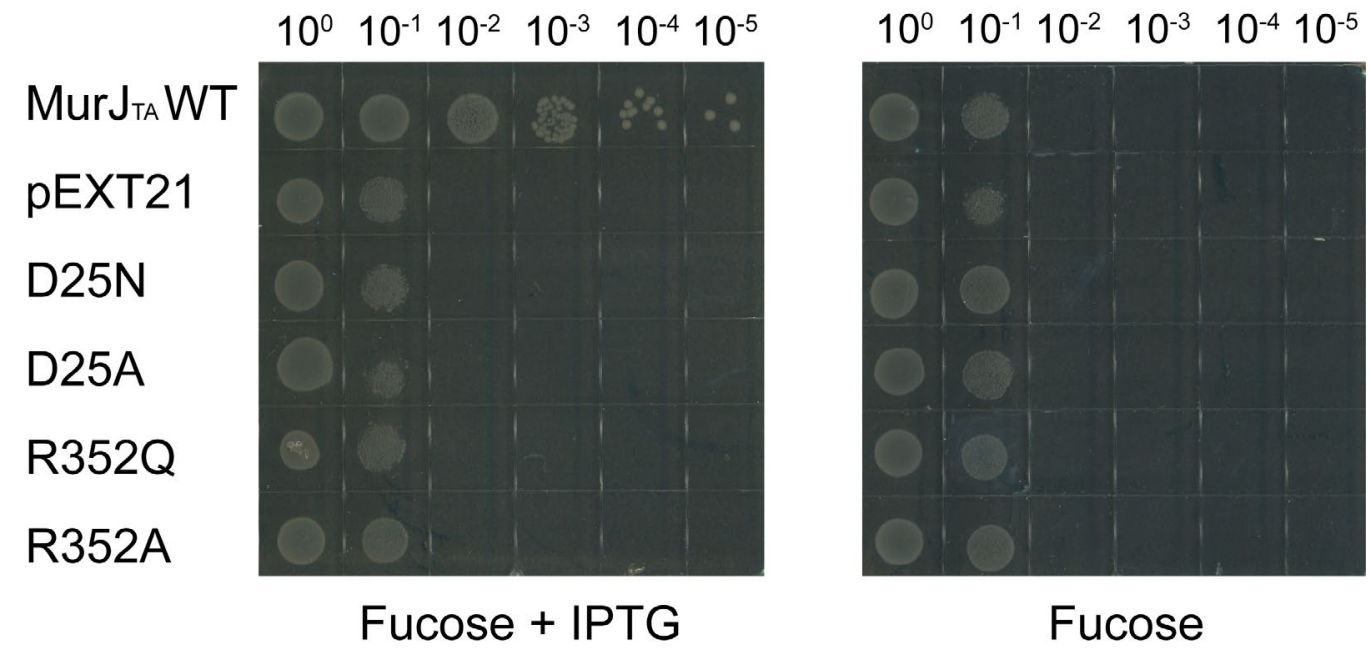

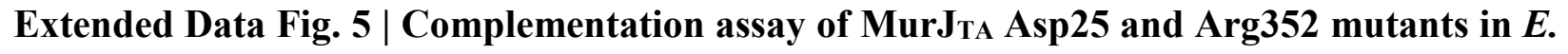

coli NR1154. Cells transformed with plasmids encoding $\mathrm{MurJ}_{\mathrm{TA}}$ (wild-type or mutant) or without insert (pEXT21) were depleted of endogenous E. coli MurJ by serial dilution on plates containing the anti-inducer D-fucose. MurJ $\mathrm{J}_{\mathrm{TA}}$ expression was induced by addition of IPTG. Data shown are representative of three biological replicates. 

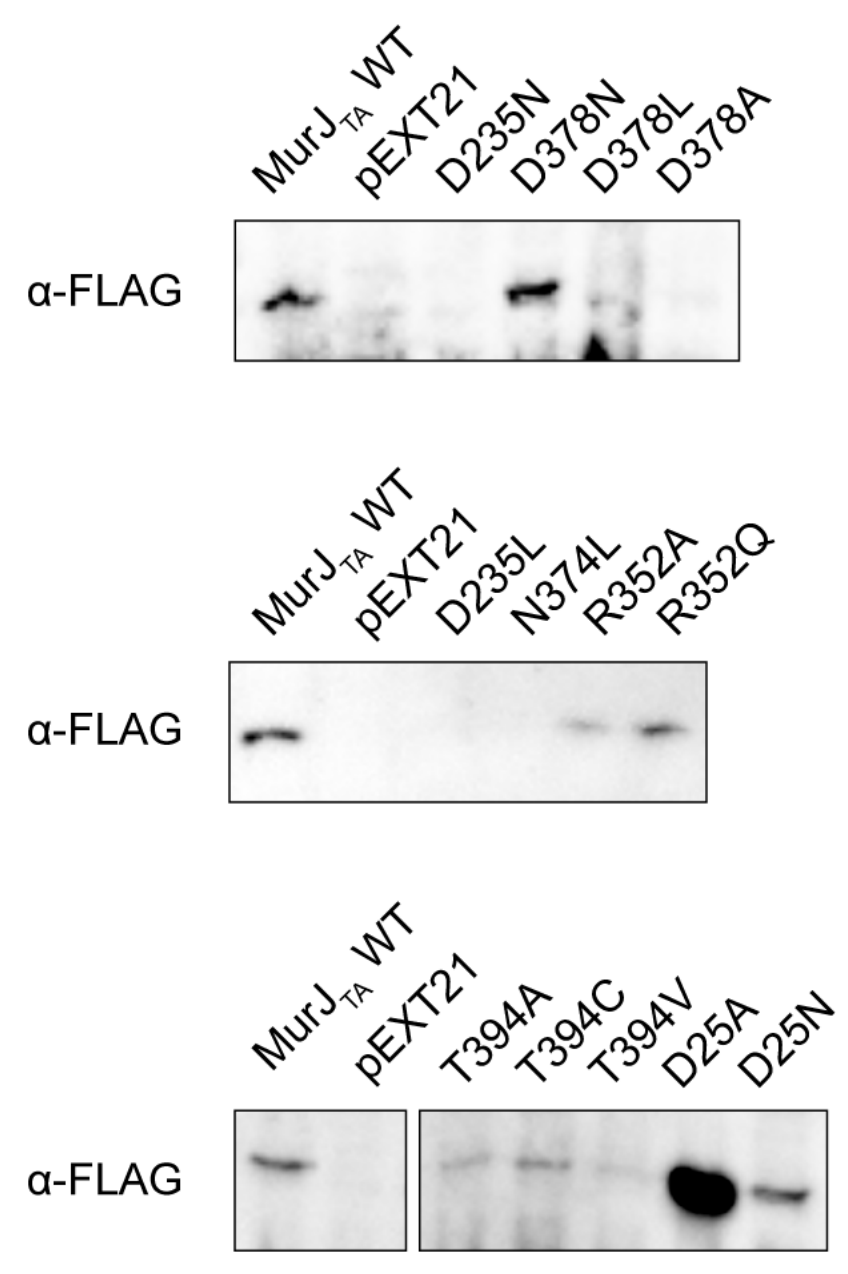

Extended Data Fig. 6 | Western blot analysis of MurJTA mutants. MurJ $\mathrm{J}_{\mathrm{TA}}$ proteins carrying a C-terminal FLAG tag were expressed by the addition of $0.1 \mathrm{mM}$ IPTG in the presence of arabinose. Total membrane fractions were isolated by ultracentrifugation and probed by mouse anti-FLAG primary antibody and horseradish peroxidase-conjugated anti-mouse secondary antibody. 
a

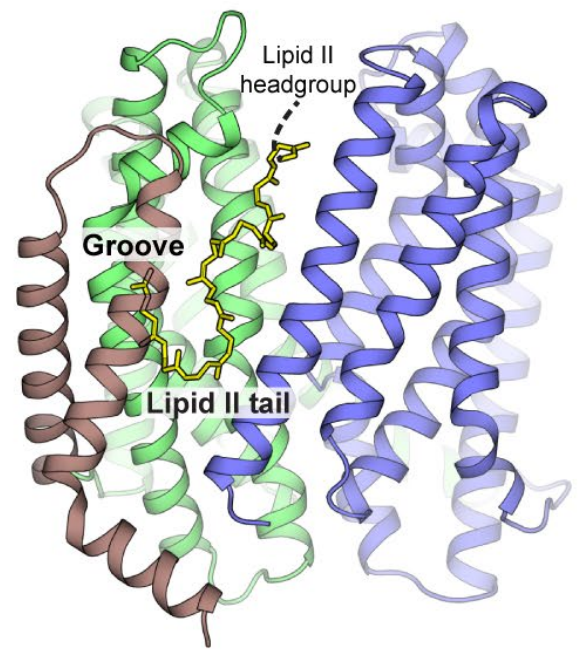

C

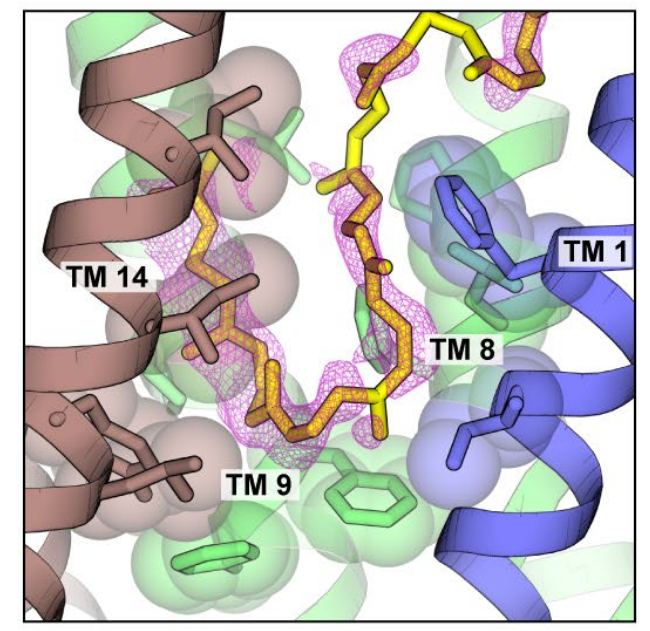

b

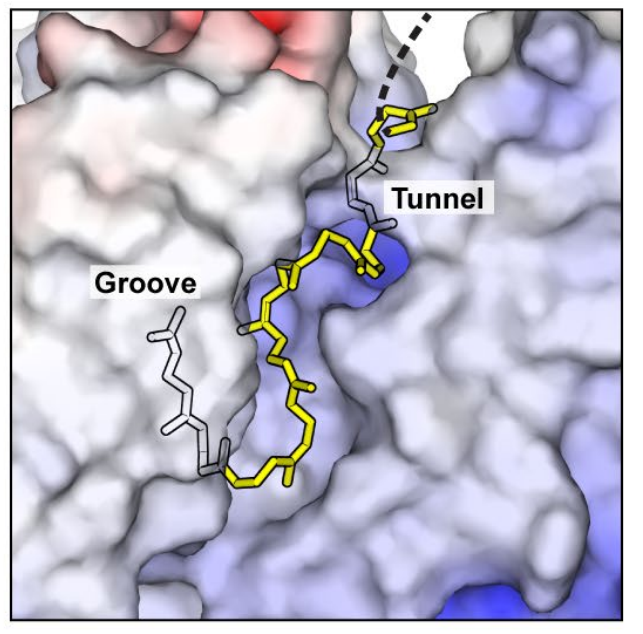

Tunnel

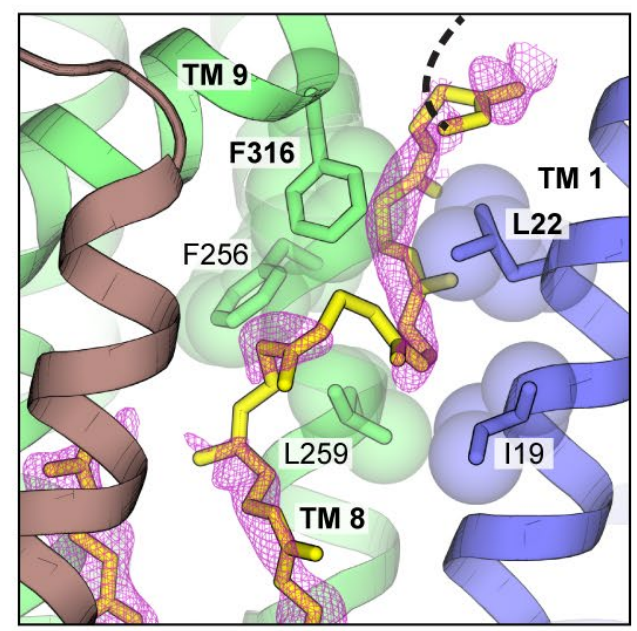

\section{Extended Data Fig. 7 | Structural features of the putative lipid-binding site in the outward}

state. a, Putative lipid-binding site. Yellow sticks denote a model of the undecaprenyl tail of Lipid II, which was never used for structure refinement or map calculation. b, The lipid is restrained by the hydrophobic groove and a short tunnel near the periplasmic side. c-d, Unmodeled 2Fo - Fc electron density peaks (purple mesh, contoured to $0.7 \sigma$ ) were observed in the hydrophobic groove (c), or in the tunnel (d) which is formed by hydrophobic residues Ile19, Leu22, Phe256, Leu259, and Phe316. 


\section{a}

IOn $\frac{\mathrm{A} 29 \mathrm{C}}{\mathrm{NMDG}} \frac{\mathrm{Na}}{-+} \frac{\mathrm{N} 4 \mathrm{C}}{-+} \frac{\mathrm{S} 113 \mathrm{C}}{-+} \frac{\mathrm{Na}}{-+} \frac{\mathrm{NMDG} \mathrm{Na}}{-+}$
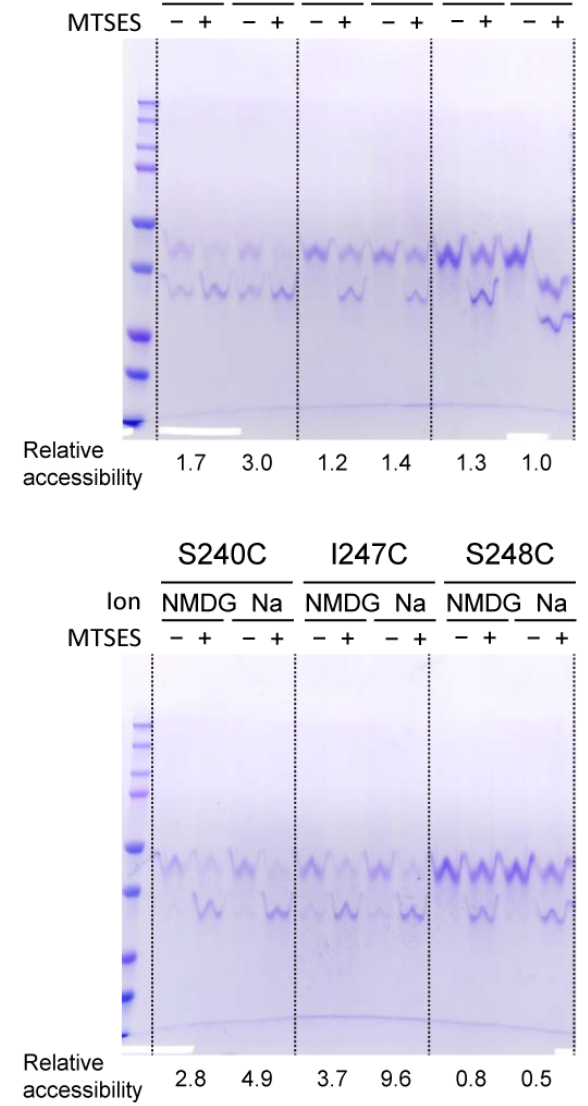

b

Fold change of accessibility

(in Na over in NMDG)

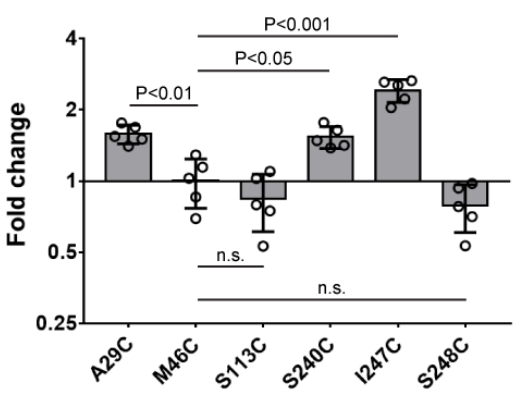

C

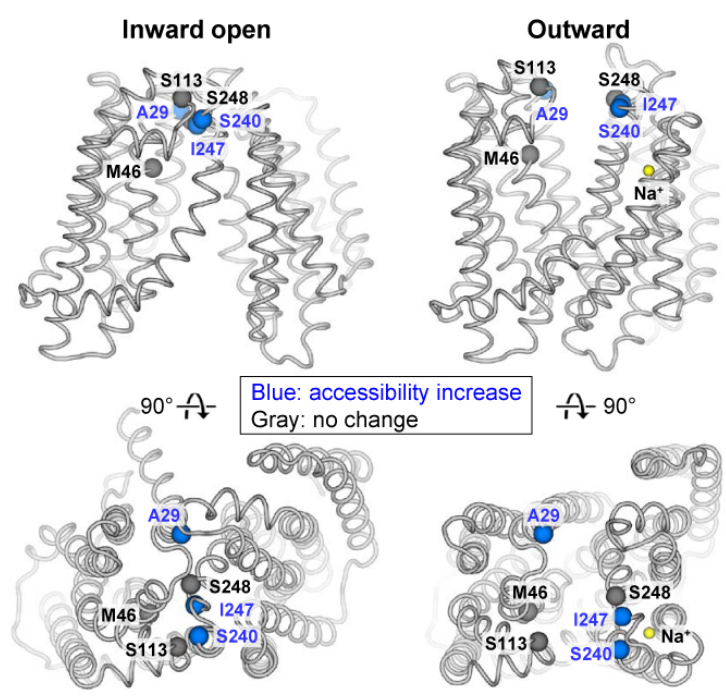

Extended Data Fig. 8 | Accessibility profile of MurJ $\mathrm{TA}_{\mathrm{TA}}$ in $\mathrm{Na}^{+}$or in $\mathrm{NMDG}^{+}$by inverse

labeling. a, Purified single cysteine mutants of MurJ $\mathrm{J}_{\mathrm{TA}}$ (no endogenous cysteines) were probed for accessibility in DDM micelles by the inverse labeling strategy in $150 \mathrm{mM}$ sodium acetate versus in $150 \mathrm{mM}$ N-methyl-D-glucamine acetate $\left(\mathrm{NMDG}^{+}\right)$. Cysteines that were not accessible to $100 \mu \mathrm{M}$ 2-sulfonatoethyl methanethiosulfonate (MTSES) would be labeled by PEG5000maleimide (PEG-Mal), resulting in a mass shift up the gel. Relative accessibility was determined as detailed in Methods, with a larger number corresponding to higher accessibility. b, Fold change in cysteine accessibility in $\mathrm{Na}^{+}$versus in NMDG $(\mathrm{n}=5$, error bars denote s.d.). Fold changes were compared to that for M46C, and P-values were calculated by paired two-tailed 

not certified by peer review) is the author/funder. All rights reserved. No reuse allowed without permission.

Student's $t$-test (abbreviation n.s. denotes not significant). c, Mapping of these positions to the inward-open and outward-facing crystal structures of MurJ $\mathrm{TA}_{\mathrm{TA}}$. 
a MurJ Complementation Growth Curve

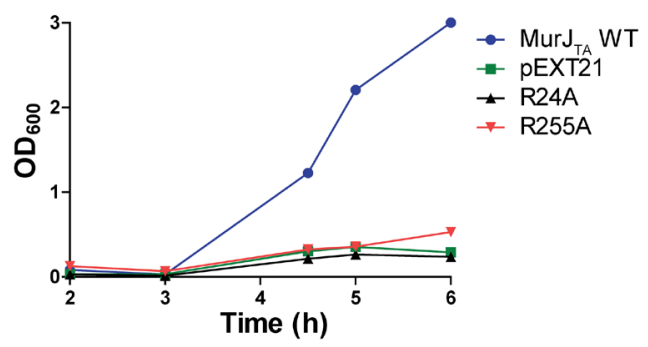

b
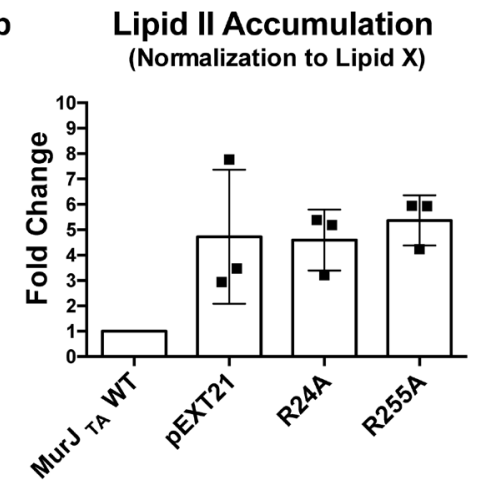

Lipid II Accumulation

(Normalization to PE)

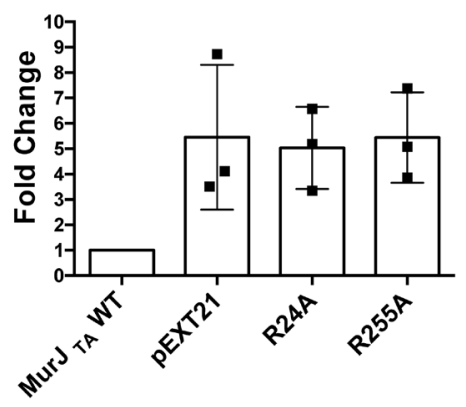

Lipid II Accumulation (Normalization to $\mathrm{OD}_{600}$ )

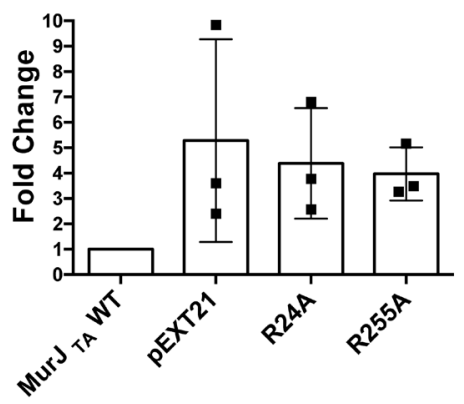

Extended Data Fig. 9 | Mass spectrometry assay of cellular Lipid II accumulation. a,

Growth curve of E. coli MurJ-depletion strain NR1154 expressing MurJ $\mathrm{TA}_{\mathrm{TA}}$ wild-type (WT), mutants (R24A/R255A), or empty vector (pEXT21). Cultures were supplemented with D-fucose to deplete endogenous E. coli MurJ. At 3 hours post-inoculation, MurJ $\mathrm{J}_{\mathrm{TA}}$ expression was induced with IPTG, and growth was monitored by optical density $\left(\mathrm{OD}_{600}\right)$. b, At 6 hours post-inoculation, cells were harvested and cellular Lipid II levels were analyzed by mass spectrometry. Cellular Lipid II levels were normalized against either Lipid X, phosphatidylethanolamine (PE), or cell density $\left(\mathrm{OD}_{600}\right)$. Lipid $\mathrm{X}$ is an intermediate in lipopolysaccharide biosynthesis pathway that is separate from the peptidoglycan synthesis pathway, but has similar chemical properties as Lipid II. Normalized Lipid II level of each replicate was divided by that of the wild-type to obtain the fold change. Data are mean \pm s.d., $n=3$ biological replicates. 